\title{
Convolution Type Operators with Symmetry in Bessel Potential Spaces ${ }^{\dagger}$
}

\author{
Luís Pinheiro de Castro and Frank-Olme Speck
}

Dedicated to Roland Duduchava on the occasion of his 70th birthday

\begin{abstract}
Convolution type operators with symmetry appear naturally in boundary value problems for elliptic PDEs in symmetric or symmetrizable domains. They are defined as truncations of translation invariant operators in a scale of Sobolev-like spaces that are convolutionally similar to subspaces of even or odd functionals. The present class, as a basic example, is closely related to the Helmholtz equation in a quadrant, where a possible solution is "symmetrically" extended to a half-plane. Explicit factorization methods allow the representation of resolvent operators in closed analytic form for a large class of boundary conditions including the two-impedance and the oblique derivative problems. Moreover they allow fine results on the regularity and asymptotic behavior of the solutions.
\end{abstract}

Mathematics Subject Classification (2010). Primary 47B35; Secondary 35J25, 45E10, 47A53, 47A68.

Keywords. Convolution type operator, symmetry, factorization, boundary value problem, quadrant, diffraction, explicit solution, Sobolev space.

\section{Introduction}

Let $r, s \in \mathbb{R}$. We consider operators of the form

$$
T=r_{+} A_{\Phi}: H^{r, c}(\mathbb{R}) \rightarrow H^{s}\left(\mathbb{R}_{+}\right)
$$

where $r_{+}$denotes the restriction operator to $\mathbb{R}_{+}, A_{\Phi}=\mathcal{F}^{-1} \Phi \cdot \mathcal{F}: H^{r} \rightarrow H^{s}$ stands for a convolution (translation invariant) operator that is invertible of order $r-s$, i.e.,

$$
\lambda^{s-r} \Phi \quad \in \quad \mathcal{G} L^{\infty}(\mathbb{R})
$$

† Accepted author's manuscript (AAM) published in [Operator Theory: Advances and Applications 258 (2017), 21-49] [DOI: 10.1007/978-3-319-47079-5_2] The final publication is available at Springer via http://link.springer.com/chapter/10.1007/978-3-319-47079-5_2 
where $\lambda(\xi)=\left(\xi^{2}+1\right)^{1 / 2}, \xi \in \mathbb{R}, \mathcal{G} L^{\infty}(\mathbb{R})$ denotes the group of invertible elements in $L^{\infty}(\mathbb{R})$ and $\mathcal{F}$ denotes the one-dimensional Fourier transformation (we also call the Fourier symbol $\Phi$ to be invertible of order $r-s$ or briefly $r-s$-invertible in this case). Moreover $H^{r}=H^{r}(\mathbb{R}), H^{s}\left(\mathbb{R}_{+}\right)$denote the common (fractional) Sobolev or Bessel potential spaces and $H^{r, c}(\mathbb{R})$ is a subspace of $H^{r}$ functionals that is convolutionally similar to the subspace of even functionals $H^{r, e}(\mathbb{R})$ or of odd functionals $H^{r, o}(\mathbb{R})$, i.e.,

$$
H^{r, c}(\mathbb{R})=P^{r, c} H^{r}=S^{-1} P^{e / o} S H^{r} \subset H^{r}
$$

where $S=A_{\Psi}$ is invertible of a certain order $q \in \mathbb{R}$ and

$$
P^{e / o}=\frac{1}{2}(I \pm J) \quad, \quad J f(x)=f(-x), x \in \mathbb{R} .
$$

Operators (1.1) are referred to as convolution type operators with symmetry (CTOS). They appear in certain applications, briefly discussed in Section 7. There we meet more particular Fourier symbols, finitely generated by symbols of differential operators, which are rational functions, and the square root of the Helmholtz operator symbol, traditionally denoted by $t(\xi)=\left(\xi^{2}-k^{2}\right)^{1 / 2}$ with a wave number $k$ with positive imaginary part and a vertical branch cut connecting $k$ and $-k$ via infinity. This leads to the fact that the "lifted" Fourier symbols (see Section 2) of the operators in question are Hölder continuous with respect to the two-point compactification $\ddot{\mathbb{R}}=\mathbb{R} \cup\{+\infty\} \cup\{-\infty\}$ and such that (in the normal case)

$$
\lambda^{s-r} \Phi, \lambda^{-q} \Psi \quad \in \quad \mathcal{G} C^{\mu}(\ddot{\mathbb{R}})
$$

where $\mu \in] 0,1[$. Therefore, the constructive part of this paper will be carried out for symbols from this class.

In the special case of $\Psi \equiv 1$ and $r=s=0$, i.e., $P^{r, c}=P^{e}$ or $P^{r, c}=P^{o}$, the operator $T$ is equivalent to a Wiener-Hopf plus/minus Hankel operator

$$
W \pm H \quad=\quad r_{+} A_{\Phi}(I \pm J) \ell_{0} \quad: \quad L^{2}\left(\mathbb{R}_{+}\right) \rightarrow L^{2}\left(\mathbb{R}_{+}\right)
$$

where several of the present results are known or easily obtained from the existing literature such as [3, 4, 10, 16. In particular the direct factorization method was obtained in [10] including small regularity results for $W \pm H: H^{r}\left(\mathbb{R}_{+}\right) \rightarrow H^{s}\left(\mathbb{R}_{+}\right)$ where $r \in]-1 / 4,3 / 4[$ or ] $-3 / 4,1 / 4[$ depending on the \pm sign. Namely, the even and odd extension operators $\ell^{e}=(I+J) \ell_{0}: H^{r}\left(\mathbb{R}_{+}\right) \rightarrow H^{r}(\mathbb{R})$ and $\ell^{o}=(I-J) \ell_{0}$ : $H^{r}\left(\mathbb{R}_{+}\right) \rightarrow H^{r}(\mathbb{R})$, respectively, are continuous and left invertible by restriction $r_{+}$if and only if $r$ belongs to the mentioned interval (see [10, Section 2). Here we turn to consider general orders $r, s \in \mathbb{R}$ by choosing $H^{r, c}(\mathbb{R})$ as domain of $T$.

A crucial point is that the projectors $P^{e / o}$ in 1.4 are continuous in all spaces $H^{s}=H^{s}(\mathbb{R}), s \in \mathbb{R}$. Sometimes we need to indicate the space order writing

$$
\begin{aligned}
& P^{s, e / o}: H^{s} \rightarrow H^{s, e / o}=\left\{u \in H^{s}: J u= \pm u\right\} \\
& \operatorname{dom} P^{s, e / o}=H^{s}, \quad \operatorname{im} P^{s, e / o}=H^{s, e / o} .
\end{aligned}
$$


However, the projectors $\ell^{e} r_{+}, \ell^{o} r_{+}$are needed, as well, exactly in the spaces of orders mentioned before, namely in so-called intermediate spaces that play a deciding role in our factorization method, because they project onto spaces with symmetry along the space $H_{-}^{s}=\left\{u \in H^{s}: \operatorname{supp} u \subset \overline{\mathbb{R}_{-}}\right\}$(in contrast to $P^{e / o}$ ).

The main results are summarized in the following:

Theorem 1.1. Let $T$ be given by $(1.1)$ with the assumptions $1.2-(1.4)$. Further let

$$
\begin{aligned}
\Upsilon_{0}=\lambda_{-}^{s} \Upsilon \lambda^{q-r} & =\lambda_{-}^{s} \Phi \Psi^{-1} \lambda^{q-r} \quad \in \quad \mathcal{G} C^{\mu}(\ddot{\mathbb{R}}) \\
\omega & =\frac{1}{2 \pi i} \int_{\mathbb{R}} d \log \Upsilon_{0}
\end{aligned}
$$

where $\lambda_{-}(\xi)=\xi-i, \xi \in \mathbb{R}$.

I. The following conditions are equivalent:

(i) $T$ is normally solvable,

(ii) $T$ is Fredholm,

(iii) $T$ is one-sided invertible,

(iv) $\Re e(\omega) \pm \frac{1}{4} \notin \mathbb{Z}$, respectively, corresponding with the e/o case,

(v) $\Upsilon$ admits a so-called "asymmetric generalized factorization" (see Section 3).

II. In the last case, a formula for a generalized inverse of $T$ is given in terms of the factorization, which can be obtained explicitly in closed analytic form.

III. In the opposite case, $T$ can be normalized by "minimal image normalization" (see Section 5) replacing the image space by a dense subspace (with continuous embedding) such that the normalized operator gains properties I (i)-(iii) and II.

The proof of this main theorem is divided into several parts, prepared and presented in the following sections $2-5$ (together with further details). Part III is of particular interest in applications. It often appears in the most important weak formulations of basic boundary value problems (looking for $H^{1}$ solutions) where boundary conditions of mixed type are given 33 or where the boundary contains conical points, see 10, for instance. These problems are often normalized (to become well-posed or Fredholm) by imposing certain "compatibility conditions" between the given data and changing the data space topology consequently. This normalization method is here directly reflected by the so-called "minimal image normalization" of the derived CTOS. It moreover helps to discover suitable compatibility conditions in complicated situations (see examples in Section 7). The method was introduced for Wiener-Hopf operators in [29] and for CTOS in [10] in the case of $r=s=0$ and $\Psi \equiv 1$, hence extended here for CTOS of the form (1.1).

Section 6 is devoted to questions of regularity of the solutions of operator equations in the sense that the solution belongs to a Bessel potential space of higher order. This is particularly interesting for the applications briefly considered in Section 7.

Finally it should be mentioned that the exposition of this article focuses a standard situation considered as a prototype of possible applications. Various generalizations and open problems are addressed in the last section. 


\section{Lifting and shifting}

We say that two bounded linear operators in Banach spaces $T$ and $U$ are equivalent, if $T=E U F$ with linear homeomorphisms $E$ and $F$. It is clear that equivalent operators are simultaneously invertible or Fredholm and (generalized) inverses can be computed from each other provided $E, F$ and their inverses are known.

Let us recall the well-known Bessel potential operators given by

$$
\begin{aligned}
& \Lambda^{s}=A_{\lambda^{s}}=\mathcal{F}^{-1} \lambda^{s} \cdot \mathcal{F} \quad: \quad H^{r} \rightarrow H^{r-s} \\
& \Lambda_{ \pm}^{s}=A_{\lambda_{ \pm}^{s}}=\mathcal{F}^{-1} \lambda_{ \pm}^{s} \cdot \mathcal{F}: \quad H^{r} \rightarrow H^{r-s}
\end{aligned}
$$

where $\lambda(\xi)=\left(\xi^{2}+1\right)^{1 / 2}, \lambda_{ \pm}(\xi)=\xi \pm i$ and $r, s \in \mathbb{R}$, cf. [13, 19, 29]. The last two have the following mapping properties (or support invariance properties):

$$
\begin{aligned}
& \Lambda_{+}^{s} H_{+}^{r}=H_{+}^{r-s}, \\
& \Lambda_{-}^{s} H_{-}^{r}=H_{-}^{r-s},
\end{aligned}
$$

$H_{ \pm}^{r}$ being the $H^{r}$ subspaces of functionals supported on $\overline{\mathbb{R}_{ \pm}}$. In terms of operator identities: If $P_{1}^{(s)}$ is a projector in $H^{s}$ onto $H_{+}^{s}$ and $P_{2}^{(s)}$ is a projector in $H^{s}$ along $H_{-}^{s}(s \in \mathbb{R})$, then we have

$$
\begin{array}{ll}
\Lambda_{+}^{s} P_{1}^{(r)} & =P_{1}^{(r-s)} \Lambda_{+}^{s} P_{1}^{(r)}, \\
P_{2}^{(r-s)} \Lambda_{-}^{s} & =P_{2}^{(r-s)} \Lambda_{-}^{s} P_{2}^{(r)} .
\end{array}
$$

Also $\Lambda^{s}$ has invariance properties according to its even symbol, which can be interpreted analogously:

Lemma 2.1. For $r, s \in \mathbb{R}$ and $P^{r, c}$ given by $(1.3$ the following holds:

$$
\begin{aligned}
\Lambda^{s} H^{r, e / o} & =H^{r-s, e / o} \\
\Lambda^{s} P^{r, c} & =P^{r-s, c} \Lambda^{s} P^{r, c} .
\end{aligned}
$$

Proof. This is a consequence of the fact that $\Lambda^{s}$ and $P^{e / o}$ interchange (taking into account convenient space orders), in combination with the facts that translation invariant operators commute and that the Fourier transformation commutes with the reflection operator $J$ and therefore with the projectors $P^{e / o}$, as well.

Theorem 2.2. Let $T$ be given by (1.1) and $\Phi_{0}=\lambda_{-}^{s} \Phi \lambda^{-r}, \Upsilon=\Phi \Psi^{-1}, \Upsilon_{0}=$ $\lambda_{-}^{s} \Upsilon \lambda^{q-r}$. The following four operators are equivalent:

$$
\begin{aligned}
& T=r_{+} A_{\Phi} \quad: H^{r, c}(\mathbb{R}) \rightarrow H^{s}\left(\mathbb{R}_{+}\right), \\
& T_{0}=r_{+} A_{\Phi_{0}}: H^{0, c}(\mathbb{R}) \rightarrow H^{0}\left(\mathbb{R}_{+}\right), \\
& U: r_{+} A_{\Upsilon}: H^{r-q, e / o}(\mathbb{R}) \rightarrow H^{s}\left(\mathbb{R}_{+}\right), \\
& U_{0}=r_{+} A_{\Upsilon_{0}}: H^{0, e / o}(\mathbb{R}) \rightarrow H^{0}\left(\mathbb{R}_{+}\right) .
\end{aligned}
$$

Herein the case e/o corresponds with the case in 1.3$). H^{0, e}\left(\mathbb{R}_{+}\right)$coincides with $L^{2, e}\left(\mathbb{R}_{+}\right)$etc. 
Proof. For systematic calculations it is convenient to argue that each of the four operators is equivalent to an operator that has the form of a general Wiener-Hopf operator [12, 32. For instance

$$
T=\left.r_{+} A_{\Phi} \sim P_{2}^{(s)} A_{\Phi}\right|_{\operatorname{im} P^{r, c}}: H^{r, c}(\mathbb{R}) \rightarrow \operatorname{im} P_{2}^{(s)},
$$

in brief $T \sim W=\left.P_{2} A\right|_{P_{1} X}$ where $A \in \mathcal{L}(X, Y), X=H^{r}, Y=H^{s}, P_{1}=$ $P^{r, c}, P_{2}=P_{2}^{(s)}$.

Now the preceding formulas imply

$$
\begin{aligned}
T & \left.\sim P_{2}^{(s)} A_{\Phi}\right|_{\operatorname{im} P^{r, c}} \\
& \left.\sim P_{2}^{(0)} \Lambda_{-}^{s} P_{2}^{(s)} A_{\Phi} P^{r, c} \Lambda^{-r}\right|_{\operatorname{im} P^{0, c}} \\
& =\left.P_{2}^{(0)} \Lambda_{-}^{s} A_{\Phi} \Lambda^{-r}\right|_{\operatorname{im} P^{0, c}} \\
& =\left.P_{2}^{(0)} A_{\Phi_{0}}\right|_{\operatorname{im} P^{0, c}} \\
& \sim T_{0} .
\end{aligned}
$$

Furthermore

$$
\begin{aligned}
T & \left.\sim P_{2}^{(s)} A_{\Phi}\right|_{\mathrm{im} P^{r, c}} \\
& \left.\sim P_{2}^{(s)} A_{\Phi} S^{-1}\right|_{\mathrm{im} P^{r-q, e / o}} \\
& =\left.P_{2}^{(s)} A_{\Phi} A_{\Psi}^{-1}\right|_{\mathrm{im} P^{r-q, e / o}} \\
& =\left.P_{2}^{(s)} A_{\Phi \Psi-1}\right|_{\mathrm{im} P^{r-q, e / o}} \\
& \sim U \\
& =r_{+} \Lambda_{-}^{-s} \ell U_{0} \Lambda^{r-q} \\
& \sim U_{0},
\end{aligned}
$$

where $\ell$ denotes any extension from $H^{s}\left(\mathbb{R}_{+}\right)$to $H^{s}$. The rest of the proof is evident.

The operator $T_{0}$ is said to be the lifted operator accompanying $T$ and $U_{0}$ is called the lifted operator accompanying $U$, cf. [29] for instance and 15 . for further studies. As mentioned in the beginning of this section, it follows that all these operators are simultaneously invertible or Fredholm (with the same defect numbers) etc.

In contrast to the lifted operators we also consider the shifted operators accompanying $T_{0}$ and $U_{0}$, namely we define, for any $\delta \in \mathbb{R}$

$$
\begin{aligned}
& T_{\delta}=\left\{\begin{array}{l}
\operatorname{Rst} T_{0}: H^{\delta, c}(\mathbb{R}) \rightarrow H^{\delta}\left(\mathbb{R}_{+}\right), \delta>0 \\
\operatorname{Ext} T_{0}: H^{\delta, c}(\mathbb{R}) \rightarrow H^{\delta}\left(\mathbb{R}_{+}\right), \delta<0
\end{array}\right. \\
& U_{\delta}=\left\{\begin{array}{l}
\operatorname{Rst} U_{0}: H^{\delta, c}(\mathbb{R}) \rightarrow H^{\delta}\left(\mathbb{R}_{+}\right), \delta>0 \\
\operatorname{Ext} U_{0}: H^{\delta, c}(\mathbb{R}) \rightarrow H^{\delta}\left(\mathbb{R}_{+}\right), \delta<0
\end{array}\right.
\end{aligned}
$$


where Rst stands for the restriction (in both domain and image) and Ext for the continuous extension of the mentioned operator to the indicated spaces, see [29] for analogous notation in a different context.

Similarly one can also define shifted operators accompanying $T$ (and $U$, respectively), needed only later in Section 6 , and briefly written in the form

$$
T^{(\delta)}=\operatorname{Rst} T: H^{r+\delta, c}(\mathbb{R}) \rightarrow H^{s+\delta}\left(\mathbb{R}_{+}\right)
$$

if $\delta>0$ or $\operatorname{Ext} T$ if $\delta<0$ etc. It is clear that these operators are bounded. Note that Rst $T$ is unique by definition (provided $T$ maps the proposed subspace into the proposed image space), but Ext $T$ is not unique in general (see the example of zero extension $\ell_{0}$ in [18, for orders smaller than $-1 / 2$ ).

The Fredholm property of $T^{(\delta)}$ will be discussed later in case of more special symbols that admit a certain factorization.

Remark 2.3. We decided to put the operator $T$ defined in 1.1 into the center of our studies instead of the equivalent operator $U$, because of its relevance in the applications as briefly shown in Section 7 .

\section{Asymmetric generalized factorization and its consequences}

First we generalize a definition from [10] (where the order of $\Phi$ was zero). Weighted Lebesgue spaces appear such as $L^{2}(\mathbb{R}, \rho)$, the spaces of measurable functions $\phi$ : $\mathbb{R} \rightarrow \mathbb{C}$ with $\rho \phi \in L^{2}(\mathbb{R})$ where $\rho$ is a given weight function, i.e., measurable and not vanishing almost everywhere. Similarly $L^{2, e / o}(\mathbb{R}, \rho)$ and $L_{-}^{2}(\mathbb{R}, \rho)$ denote functions with $\rho \phi \in L^{2, e / o}(\mathbb{R})$ and $\rho \phi \in L_{-}^{2}(\mathbb{R})$, respectively.

Definition 3.1. An invertible symbol $\Phi$ of order $r-s$ (see (1.2) ) admits an asymmetric generalized factorization with respect to $\left(L^{2}\left(\mathbb{R}, \lambda^{r}\right), L^{2}\left(\mathbb{R}, \lambda^{s}\right), \Pi_{1}, \Pi_{2}\right)$, where $\Pi_{1} \in \mathcal{L}\left(L^{2}\left(\mathbb{R}, \lambda^{r}\right)\right)$ is a projector onto $L^{2, e / o}\left(\mathbb{R}, \lambda^{r}\right)$ and $\Pi_{2} \in \mathcal{L}\left(L^{2}\left(\mathbb{R}, \lambda^{s}\right)\right)$ is a projector along $L_{-}^{2}\left(\mathbb{R}, \lambda_{-}^{s}\right)$, in brief $A G F$ and written as

$$
\Phi=\Phi_{-} \zeta^{\kappa} \Phi_{e},
$$

if (j) $\kappa \in \mathbb{Z}, \zeta(\xi)=(\xi-i) /(\xi+i)$ for $\xi \in \mathbb{R}$, furthermore $(\mathrm{jj})$

$$
\begin{aligned}
& \Phi_{-} \in L_{-}^{2}\left(\mathbb{R}, \lambda_{-}^{s-2}\right), \quad \Phi_{-}^{-1} \in L_{-}^{2}\left(\mathbb{R}, \lambda_{-}^{-s-1}\right), \\
& \Phi_{e} \in L^{2, e}\left(\mathbb{R}, \lambda^{-r-1}\right), \quad \Phi_{e}^{-1} \in L^{2, e}\left(\mathbb{R}, \lambda^{r-2}\right) \\
& \Phi_{-} \in L_{-}^{2}\left(\mathbb{R}, \lambda_{-}^{s-1}\right), \quad \Phi_{-}^{-1} \in L_{-}^{2}\left(\mathbb{R}, \lambda_{-}^{-s-2}\right), \\
& \Phi_{e} \in L^{2, e}\left(\mathbb{R}, \lambda^{-r-2}\right), \quad \Phi_{e}^{-1} \in L^{2, e}\left(\mathbb{R}, \lambda^{r-1}\right) \text { for the "o-case" }
\end{aligned}
$$

and finally (jjj) the operator composed by multiplication operators and the Hilbert transform $S_{\mathbb{R}}=\mathcal{F}^{-1} \operatorname{sgn}(\cdot) \cdot \mathcal{F}$

$$
V=\Phi_{e}^{-1} \cdot S_{\mathbb{R}} \zeta^{-\kappa} \cdot S_{\mathbb{R}} \Phi_{-}^{-1} \cdot \quad: \quad L_{+}^{2}\left(\mathbb{R}, \lambda^{s}\right) \rightarrow L^{2}\left(\mathbb{R}, \lambda^{r}\right)
$$

is bounded (as a composition of unbounded operators, extended from a dense subspace). The number $\kappa$ in (3.1) is called factorization index of the AGF of $\Phi$. 
As in the case of Toeplitz and Wiener-Hopf operators 4, 10, 23 the factor spaces (to which $\Phi_{-}$etc. belong) are the closures of the spaces of sufficiently decreasing rational functions without poles in the closed lower half-plane $\overline{\mathbb{C}_{-}}=$ $\{\xi \in \mathbb{C}: \Im m(\xi) \leq 0\}$ or of those which are even, respectively, due to the weighted $L^{2}$ norm.

For convenience we put $A_{e}=\mathcal{F}^{-1} \Phi_{e} \cdot \mathcal{F}, A_{-}=\mathcal{F}^{-1} \Phi_{-} \cdot \mathcal{F}$ and $C=\mathcal{F}^{-1} \zeta^{\kappa} \cdot \mathcal{F}$. In general $A_{e}: H^{r} \rightarrow L^{2}$ and $A_{-}: L^{2} \rightarrow H^{s}$ are unbounded operators whilst $C: L^{2} \rightarrow L^{2}$ is bounded. The domains of the factors are such that

$$
A_{\Phi}=A_{-} C A_{e}: \mathcal{D}_{1} \rightarrow \mathcal{D} \rightarrow \mathcal{D} \rightarrow \mathcal{D}_{2}
$$

splits in the sense of bijective mappings where

$$
\begin{aligned}
& \mathcal{D}_{1}=\mathcal{D}\left(A_{e}\right) \\
& \mathcal{D}_{2}=\mathcal{D}\left(A_{-}^{-1}\right) \\
& \mathcal{D}=\left.\operatorname{im} A_{e}\right|_{\mathcal{D}_{1}}=\left.\operatorname{im} A_{-}^{-1}\right|_{\mathcal{D}_{2}},
\end{aligned}
$$

$\mathcal{D}_{1}, \mathcal{D}_{2}$ and $\mathcal{D}$ are dense in $L^{2}$ and the restrictions of $C^{ \pm 1}, \ell^{e / o} r_{+}: \mathcal{D} \rightarrow \mathcal{D}$ are well-defined.

Remark 3.2. An AGF (3.1) defines a Wiener-Hopf factorization through the intermediate space (FIS) $Z=L^{2}(\mathbb{R})$ in the sense of [36]. The resulting factorization (3.4) defines a FIS, as well. That means, in a general setting, a boundedly invertible linear operator $A \in \mathcal{L}(X, Y)$ in Banach spaces $X, Y$ admits a FIS with respect to $X, Y$ and two projectors $P_{1} \in \mathcal{L}(X)$ and $P_{2} \in \mathcal{L}(Y)$ if

$$
\begin{aligned}
A & =\quad A_{-} \quad C \quad A_{+} \\
& : \quad Y \leftarrow Z \leftarrow Z \leftarrow X
\end{aligned}
$$

where the factors $A_{ \pm}$and $C$ possess the following properties: They are linear and boundedly invertible in the above setting with an additional Banach space $Z$ called intermediate space. Further there is a projector $P \in \mathcal{L}(Z)$ such that

$$
A_{+} P_{1} X=P Z \quad, \quad A_{-} Q Z=Q_{2} Y
$$

with $Q=I_{Z}-P$ and such that $C \in \mathcal{L}(Z)$ splits the space $Z$ twice into four subspaces with

$$
\begin{aligned}
Z= & \overbrace{X_{1} \dot{+} X_{0}}^{P Z} \dot{\overbrace{X_{2} \dot{+} X_{3}}^{Q Z}} \\
& =\underbrace{Y_{1}^{Y_{1}+Y_{2}}}_{P Z} \dot{\downarrow} \underbrace{Y_{0} \dot{+} Y_{3}}_{Q Z}
\end{aligned}
$$

where $C$ maps each $X_{j}$ onto $Y_{j}, j=0,1,2,3$, i.e., the complemented subspaces $X_{0}, X_{1}, \ldots, Y_{3}$ are images of corresponding projectors $p_{0}, p_{1}, \ldots, q_{3}$, namely $X_{0}=$ $p_{0} Z=C^{-1} Q C P Z, X_{1}=p_{1} Z=C^{-1} P C P Z, \ldots, Y_{3}=q_{3} Z=C Q C^{-1} Q Z$. 
$A_{ \pm}$are called strong $W H$ factors and $C$ is said to be a cross factor, acting from a space $Z$ onto the same space $Z$ as a bounded linear operator. This kind of factorization avoids the use of unbounded factors.

Theorem 3.3. Let $T$ be given by $(1.1)$ and let $\Upsilon=\Phi \Psi^{-1}$ admit an AGF with respect to $P_{1}=P^{r, e / o}$ and $P_{2}=P_{2}^{(s)}$ with factorization index $\kappa \in \mathbb{Z}$. Then $T$ is Fredholm with analytical index Ind $T=-\kappa$ and a reflexive generalized inverse of $T$ is given by

$$
\begin{array}{r}
T^{-}=\left.P^{r-q, c} S^{-1} V\right|_{\operatorname{im} P_{2}^{(s)}}=\left.S^{-1} P^{e / o} A_{e}^{-1} \ell^{c} r_{+} C^{-1} \ell^{c} r_{+} A_{-}^{-1}\right|_{\operatorname{im} P_{2}^{(s)}} \\
=\left.A_{\Psi^{-1}} \frac{1}{2}(I \pm J) A_{e}^{-1} \ell^{c} r_{+} A_{\zeta^{-\kappa}} \ell^{c} r_{+} A_{-}^{-1}\right|_{\operatorname{im} P_{2}^{(s)}}
\end{array}
$$

where $\ell^{c}$ stands for $\ell^{e / o}$ in the corresponding case of $P^{r, e / o}$, respectively. Moreover $T$ is one-sided invertible and its defect numbers read

$$
\alpha(T)=\operatorname{dim} \operatorname{ker} T=\max \{0,-\kappa\}, \beta(T)=\operatorname{codim} \operatorname{im} T=\max \{\kappa, 0\} .
$$

Proof. Note that $\ell^{c} r_{+}=\ell^{e / o} r_{+}$and $P^{e / o}$ have the same images, but different kernels (in $L^{2}$ and in $H^{\delta},|\delta|<1 / 2, r_{+}$being bounded and left invertible by $\left.\ell_{0}\right)$. It is important that $\ell^{c} r_{+}$maps onto $L^{2, e / o}$ along $L_{-}^{2}$ (and not along $L^{2, o / e}$ ). With the techniques of Section 2 it is not difficult to verify that $T T^{-} T=T$ and $T^{-} T T^{-}=T^{-}$, i.e., that 3.9 represents a reflexive generalized inverse of $T$ (cf. details in the proof of Theorem 3.2 in [10] for the case of $r=s=0$ ). Further $T \sim U_{0}$ by 2.8), 2.9) for which the remaining results are known [10].

Corollary 3.4. The operators $T, T_{0}, U, U_{0}$ have all the same properties mentioned in Theorem 1.1. There kernels and complements of their images are explicitly represented by use of the formulas in Section 2 and in [10], Section 4: For instance, if $\kappa<0$ :

$$
\begin{aligned}
\operatorname{ker} r_{+} C \ell^{e} r_{+} & =\mathcal{F}^{-1} \operatorname{span}\left\{\lambda^{-2}, \lambda^{-4}, \ldots, \lambda^{2 \kappa}\right\} \\
& =\ell^{e} \mathcal{F}^{-1} \operatorname{span}\left\{\lambda_{+}^{-1}, \ldots, \lambda_{+}^{\kappa}\right\} .
\end{aligned}
$$

Remark 3.5. The choice of weights in (3.2) looks somehow arbitrary. As we see later (in Proposition 4.6), it is strongly motivated by the present symbol class (1.5) and the theory of Toeplitz operators [4, 23]. See also [10] for "small orders" of $r$ and $s$.

\section{Constructive factorization}

Constructive AGF is based upon some knowledge about the $\Phi$-factorization 23 (alias generalized factorization [31] or Wiener-Hopf factorization [4]) of bounded measurable functions $\phi \in \mathcal{G} L^{\infty}$ in $L^{2}$ and the equivalence of an AGF of $\psi$ with another kind of factorization, the so-called antisymmetric factorization [3, 10, 16. of a related function $G=\psi \widetilde{\psi}^{-1}$. To this end we need two well-known definitions. 
Definition 4.1. A function $\psi \in \mathcal{G} L^{\infty}$ admits a $\Phi$-factorization in $L^{2}$ if

$$
\psi=\psi_{-} \zeta^{\kappa} \psi_{+}
$$

where $\kappa \in \mathbb{Z}$, furthermore

$$
\psi_{-}^{ \pm 1} \in L_{-}^{2}\left(\mathbb{R}, \lambda_{-}^{-1}\right) \quad, \quad \psi_{+}^{ \pm 1} \in L_{+}^{2}\left(\mathbb{R}, \lambda_{+}^{-1}\right),
$$

and finally

$$
V=A_{+}^{-1} \ell_{0} r_{+} A_{\zeta^{-\kappa}} \ell_{0} r_{+} A_{-}^{-1} \quad: \quad L^{2} \rightarrow L^{2}
$$

is bounded (as a composition of unbounded operators, extended from a dense subspace).

Definition 4.2. If $\kappa=0$ in one of the foregoing factorizations we call it a canonical factorization. If moreover, in a canonical $\Phi$-factorization (4.1), $\psi_{+}=\widetilde{\psi}_{-}^{-1}$ where $\widetilde{\psi_{-}}=J \psi_{-}$, this factorization is said to be antisymmetric.

Now we are in the position to carry out the effective factorization of invertible scalar symbols which are most relevant in the applications mentioned before, namely elements of the algebra of Hölder continuous functions on $\ddot{\mathbb{R}}=[-\infty,+\infty]$ :

$$
\begin{aligned}
C^{\mu}(\ddot{\mathbb{R}})= & \left\{\phi \in C^{\mu}(\mathbb{R}): \phi( \pm \infty)=\lim _{\xi \rightarrow \pm \infty} \phi(\xi)\right. \text { exist and } \\
& \left.\left.\phi(\xi)-\phi( \pm \infty)=\mathcal{O}\left(|\xi|^{-\mu}\right) \text { as } \xi \rightarrow \pm \infty\right\}, \quad \mu \in\right] 0,1[.
\end{aligned}
$$

In the case of $\phi(+\infty)=\phi(-\infty)$ we write $\phi \in C^{\mu}(\dot{\mathbb{R}})$. The subclasses of invertible elements are denoted by $\mathcal{G} C^{\mu}(\ddot{\mathbb{R}})$ and $\mathcal{G} C^{\mu}(\dot{\mathbb{R}})$, respectively. The following result is known from [10. We give a sketch of the proof because it contains the technical details of the method that are needed in practice.

Proposition 4.3. Let $\phi \in \mathcal{G} C^{\mu}(\ddot{\mathbb{R}})$ where $\left.\mu \in\right] 0,1[$ and define

$$
\omega=\frac{1}{2 \pi i} \int_{\mathbb{R}} d \log \phi .
$$

Further let

$$
\Re e(\omega) \pm \frac{1}{4} \notin \mathbb{Z}
$$

where the sign corresponds with the $\ell^{e} / \ell^{o}$ case. Then $\phi$ admits an AGF (see Definition (3.1) given by the following formulas:

$$
\begin{aligned}
\kappa & =\max \left\{z \in \mathbb{Z}: z \leq \Re e(\omega) \pm \frac{1}{4}\right\} \\
\psi & =\zeta^{-\omega} \phi^{-1}(+\infty) \phi \\
\widetilde{\psi} & =J \psi \\
\phi_{-} & =\lambda_{-}^{2(\omega-\kappa)} \exp \left\{P_{-} \log \left(\psi \widetilde{\psi}^{-1}\right)\right\} \\
\phi_{e} & =\zeta^{-\kappa} \phi_{-}^{-1} \phi,
\end{aligned}
$$

where $P_{-}=\frac{1}{2}\left(I-S_{\mathbb{R}}\right)$. 
Proof. We write $\phi$ in the form

$$
\phi(\xi)=\phi(+\infty)\left(\frac{\xi-i}{\xi+i}\right)^{\omega} \psi(\xi), \quad \xi \in \mathbb{R}
$$

where $\psi \in \mathcal{G} C^{\mu}(\dot{\mathbb{R}})$ with vanishing winding number. Precisely, let $\omega=\sigma+i \tau \in \mathbb{C}$ with real and imaginary parts given by

$$
\sigma=\frac{1}{2 \pi} \int_{\mathbb{R}} d \arg \phi, \quad \tau=\frac{1}{2 \pi} \log \left|\frac{\phi(-\infty)}{\phi(+\infty)}\right| .
$$

Now we follow an idea of Basor and Ehrhardt 3, 10, from the theory of Toeplitz plus Hankel operators and consider the function

$$
G=\psi \widetilde{\psi}^{-1} .
$$

It has the same properties as $\psi$ before plus the (anti-) symmetry property $\widetilde{G}^{-1}=$ $G$. Thus it admits a canonical anti-symmetric factorization

$$
G=G_{-} G_{+}=G_{-} \widetilde{G}_{-}^{-1}
$$

where $G_{ \pm} \in \mathcal{G} C_{ \pm}^{\mu}(\dot{\mathbb{R}})$.

By the help of 4.10 and 4.11 we obtain an AGF of $\psi$, putting

$$
\psi=\psi_{-} \psi_{e}=G_{-} \psi_{e} .
$$

Here $\psi_{e}$ is even since this fact is equivalent to

$$
\begin{aligned}
& \psi_{e}=\widetilde{\psi}_{e} \\
& \psi G_{-}^{-1}=\widetilde{\psi} \widetilde{G}_{-}^{-1} \\
& \psi \widetilde{\psi}^{-1}=G_{-} \widetilde{G}_{-}^{-1}
\end{aligned}
$$

which was our factorization 4.12 of 4.11). The factors of $\psi$ in 4.13 belong to $\mathcal{G} C^{\mu}(\dot{\mathbb{R}})$. Therefore $\phi$ has an AGF if and only if $\zeta^{\omega}$ admits an AGF.

Now let 4.6 be satisfied, thus we can write

$$
\omega=\sigma+i \tau=\kappa+\eta+i \tau
$$

where $\kappa \in \mathbb{Z}$ and $\eta \in]-1 / 4,3 / 4\left[\right.$ in the case $\ell^{c}=\ell^{e}$, that will be treated first. Considering

$$
\begin{aligned}
\zeta^{\omega} & =\lambda_{-}^{2(\omega-\kappa)} \zeta^{\kappa}\left(\lambda_{-} \lambda_{+}\right)^{\kappa-\omega} \\
& =\lambda_{-}^{2(\eta+i \tau)} \zeta^{\kappa} \lambda^{-2(\eta+i \tau)}
\end{aligned}
$$

we have an AGF with respect to $\ell^{e}$ : the factors belong to the spaces mentioned in (3.2) and the operator $V$ in (3.3) corresponding with 4.15) is bounded. This is a consequence of the fact that, due to 4.15,

$$
C_{\omega}=\mathcal{F}^{-1} \zeta^{\omega} \cdot \mathcal{F}=C_{-} C C_{e}: L^{2} \rightarrow H^{2 \eta} \rightarrow H^{2 \eta} \rightarrow L^{2}
$$

is a composition of boundedly invertible operators where $2 \eta \in]-1 / 2,3 / 2[$, such that $\ell^{e} r_{+}$is bounded in $H^{2 \eta}$. I.e., the factors $C_{-}, C, C_{e}$ are bijections with respect 
to the spaces mentioned in 4.16 . The combination with 4.8 and 4.12 yields an AGF of $\phi$.

The case $\ell^{c}=\ell^{o}$ runs analogously with

$$
\eta \in]-3 / 4,1 / 4[\text { and therefore } 2 \eta \in]-3 / 2,1 / 2[\text {. }
$$

Corollary 4.4. Under the same assumptions as before, the AGF of $\phi$ yields bounded operator factorizations of the multiplication operator $\phi \cdot$, of the convolution operator $A=\mathcal{F}^{-1} \phi \cdot \mathcal{F}$ and of the Wiener-Hopf plus Hankel operator $T=r_{+} A \ell^{e}$ through an intermediate space $Z$ which is a weighted $L^{2}$ space or corresponding Sobolev space, respectively. More precisely, we have a commutative diagram of bijective multiplication operators

$$
\begin{array}{ccc} 
& \phi \cdot & \\
L^{2}(\mathbb{R}) & \longrightarrow & L^{2}(\mathbb{R}) \\
\phi_{e} \cdot \downarrow & \uparrow \phi_{-} \cdot \\
L^{2}\left(\mathbb{R}, \lambda^{-2 \eta}\right) & \underset{ }{\longrightarrow} & L^{2}\left(\mathbb{R}, \lambda^{-2 \eta}\right) \\
& \zeta^{\kappa} . &
\end{array}
$$

and, correspondingly written as bounded operator factorization

$$
A=A_{-} C A_{e}: L^{2} \rightarrow H^{2 \eta} \rightarrow H^{2 \eta} \rightarrow L^{2},
$$

a so-called asymmetric factorization through an intermediate space (AFIS) 10 (this notation was used in the special case where the "plus factor" in a FIS of a convolution operator has an even symbol whilst the "minus factor" possesses the common properties, see also [7, 36]). Consequently we have a splitting of $T$ into bounded operators, as well, namely

$$
\begin{aligned}
& T=r_{+} A_{-} \ell \quad\left(r_{+} C \ell^{e}\right) \quad r_{+} A_{e} \ell^{e} \\
& : \quad L^{2}\left(\mathbb{R}_{+}\right) \leftarrow H^{2 \eta}\left(\mathbb{R}_{+}\right) \leftarrow H^{2 \eta}\left(\mathbb{R}_{+}\right) \leftarrow L^{2}\left(\mathbb{R}_{+}\right)
\end{aligned}
$$

(where the extension $\ell: H^{2 \eta}\left(\mathbb{R}_{+}\right) \rightarrow H^{2 \eta}$ is arbitrary, e.g. $\ell=\ell^{e}$ or $\ell=\ell^{o}$ ).

Furthermore the intermediate space $Z=H^{2 \eta}$ in (4.19) has the following properties, for all possible choices of $\omega$,

$$
\begin{aligned}
& C=\zeta^{\kappa} \cdot \in \mathcal{L}(Z) \quad \text { for all } \quad \kappa \in \mathbb{Z} \\
& \ell^{e} r_{+} \in \mathcal{L}(Z) .
\end{aligned}
$$

Finally, denoting the $L^{2}$ functions with rational Fourier images by $\widehat{\mathcal{R}}_{0}=\widehat{\mathcal{R}} \cap L^{2}(\mathbb{R})$, we have

$$
\ell^{e} r_{+} \widehat{\mathcal{R}}_{0} \underset{\text { dense }}{\subset} \ell^{e} r_{+} Z
$$


Theorem 4.5. Let $T$ be given by (1.1) with the assumptions 1.2 - 1.4. Further let

$$
\begin{aligned}
\Upsilon_{0}=\lambda_{-}^{s} \Upsilon \lambda^{q-r} & =\lambda_{-}^{s} \Phi \Psi^{-1} \lambda^{q-r} \quad \in \quad \mathcal{G} C^{\mu}(\ddot{\mathbb{R}}) \\
\omega & =\frac{1}{2 \pi i} \int_{\mathbb{R}} d \log \Upsilon_{0} .
\end{aligned}
$$

Then the following conditions are equivalent:

(i) $T$ is Fredholm,

(ii) $\Re e(\omega) \pm \frac{1}{4} \notin \mathbb{Z}$, respectively, corresponding with the e/o case,

(iii) $\Upsilon$ admits an $A G F$ (3.1) with respect to $P_{1}=P^{r-q, e / o}$ and $P_{2}=P_{2}^{(s)}$,

and, moreover,

(iii') $A_{\Upsilon_{0}}$ admits an AFIS 4.18 through the space $Z=H^{2 \eta}$ where $\left.2 \eta \in\right]-1 / 2,3 / 2[$ or $2 \eta \in]-3 / 2,1 / 2[$, respectively, $\eta$ being given by (4.14).

Further the factorization of $\Psi$ is unique up to constant factors in $\Psi_{-}$and $\Psi_{e}$ (inverse to each other).

Proof. From Theorem 2.2 we know that the Fredholm property of $T$ is equivalent to the Fredholm property of $U_{0}$, which corresponds with the case where $r=s=0$ and $\Psi \equiv 1$. In this special case the result is known, see [10], Proposition 4.2. Hence the equivalence of the mentioned items extends to the general situation, by application of the results of Theorem 2.2. The last statement is known from Wiener-Hopf factorization, to which the present operators are related by 4.11).

The foregoing results gave us the motivation for the definition 3.1 of an AGF. We underline this by the following:

Proposition 4.6. Let $\psi \in \mathcal{G} C^{\mu}(\ddot{\mathbb{R}})$. Then $\psi$ admits a canonical $A G F$ with $r=s=0$ (in the sense of Definition 3.1) if and only if $G=\psi \widetilde{\psi}^{-1}$ admits an antisymmetric canonical $\Phi$-factorization in $L^{2}$.

Proof. Sufficiency: Let $G=G_{-} G_{+}$be an antisymmetric canonical $\Phi$-factorization of $G=\psi \widetilde{\psi}^{-1}$, i.e., $G_{+}=G_{-}^{-1}$. Then we obtain an AGF of $\psi$ as in 4.10 4.15):

$$
\psi=\psi_{-} \psi_{e}=G_{-} \psi_{e},
$$

i.e., $\psi_{-}=G_{-}$and $\psi_{e}=G_{-}^{-1} \psi$. Hence we have to show that $\psi_{e}$ is even. This fact is equivalent to

$$
\begin{aligned}
& \psi_{e}=\widetilde{\psi_{e}} \\
& \psi G_{-}^{-1}=\widetilde{\psi}{\widetilde{G_{-}}}^{-1} \\
& \psi \widetilde{\psi}^{-1}=G_{-}{\widetilde{G_{-}}}^{-1}
\end{aligned}
$$

which is true by assumption. The factors obviously belong to the right classes.

Necessity: Let $\psi=\psi_{-} \psi_{e}$ be a canonical AGF and consider $G=\psi \widetilde{\psi}^{-1}=$ $G_{-} G_{+}$where we put $G_{-}=\psi_{-}$. Then we have to show that $G_{+}={\widetilde{G_{-}}}^{-1}$, i.e., we have an antisymmetric factorization of $G$. This follows from

$$
G_{+}=\psi_{-}^{-1} \psi \widetilde{\psi}^{-1}=\psi_{-}^{-1} \psi_{-} \psi_{e}{\widetilde{\psi_{e}}}^{-1}{\widetilde{\psi_{-}}}^{-1}=G_{-}^{-1} .
$$


Again the factors obviously belong to the right classes.

Now we are in the position to organize the proof of Theorem 1.1:

Proof of Theorem 1.1. First we see that $T \sim U_{0}$ by Theorem 2.2. Second we know from Proposition 4.6 that an AGF of the Fourier symbol $\Upsilon_{0} \in \mathcal{G} C^{\mu}(\ddot{\mathbb{R}})$ of $U_{0}$ implies a generalized factorization of $G=\Upsilon_{0} \widetilde{\Upsilon}_{0}^{-1}$ which is asymmetric (see (4.11) ) and vice versa, an asymmetric generalized factorization of $G$ implies an AGF of $\Upsilon_{0}$.

With the help of $G$ we have from [10] that the following conditions are equivalent:

(i) $U_{0}$ is normally solvable,

(ii) $U_{0}$ is Fredholm,

(iii) $U_{0}$ is one-sided invertible,

(iv) $\Re e(\omega) \pm \frac{1}{4} \notin \mathbb{Z}$, respectively, corresponding with the $e / o$ case,

(v) $\Upsilon_{0}$ admits an AGF.

Translating this back into the corresponding properties of $T$ and $\Upsilon$ by Theorem 2.2 and Definition 3.1, we come to the list of equivalent properties in Part I of Theorem 1.1.

Part II is the main content of Theorem 3.3.

Part III is a summary of the main results of the following section.

\section{Normalization}

Now we tackle the critical case of Theorem 1.1 where condition (iv) is violated and $T$ is not Fredholm in the considered space setting. First we recall two definitions from [10, 29].

Definition 5.1. Let $X, Y$ be Banach spaces, $S \in \mathcal{L}(X, Y)$ a bounded linear operator which is not normally solvable, i.e., $\operatorname{im} S$ is not closed. If there is another Banach space $Y_{1}$ such that

$$
Y_{1} \underset{\text { dense }}{\subset} Y, \quad Y_{1} \neq Y, \quad \operatorname{im} S \subset Y_{1}
$$

and

$$
\stackrel{\prec}{S}=\operatorname{Rst} S: X \rightarrow Y_{1}
$$

is normally solvable, then $\stackrel{\prec}{S}$ is called a minimal image normalization of $S$.

Definition 5.2. For any $s \in \mathbb{C}$ consider the modified Bessel potential space

$$
\stackrel{\prec}{H^{s}}\left(\mathbb{R}_{+}\right)=r_{+} A_{\lambda_{-}}^{-s-1 / 2} H_{+}^{-1 / 2}=r_{+} A_{\lambda_{-}}^{-s-1 / 2} A_{\lambda_{+}}^{1 / 2} L_{+}^{2}
$$

equipped with the norm induced by $H_{+}^{-1 / 2}$, namely by

$$
\|\psi\|_{H^{s}\left(\mathbb{R}_{+}\right)}=\left\|\ell_{0} \varphi\right\|_{H^{-1 / 2}} \quad, \quad \varphi=r_{+} A_{\lambda_{-}}^{s+1 / 2} \ell \psi
$$


where $\ell \psi \in H^{\Re e(s)}$ is any extension of $\psi \in H^{\Re e(s)}\left(\mathbb{R}_{+}\right)$.

Remark 5.3. The modified Bessel potential spaces can be seen in a similar way as the common spaces of Bessel potentials as being the images of certain Bessel potential operators:

$$
H^{s}\left(\mathbb{R}_{+}\right)=r_{+} A_{\lambda_{-}}^{-s} L_{+}^{2} \quad \text { for } \quad s \in \mathbb{R} .
$$

In the special cases of $s= \pm 1 / 2$ we have $\stackrel{\prec}{H^{s}}\left(\mathbb{R}_{+}\right)=\widetilde{H}^{ \pm 1 / 2}\left(\mathbb{R}_{+}\right)$, the dense subspaces of $H^{ \pm 1 / 2}\left(\mathbb{R}_{+}\right)$of functionals extensible by zero into $H^{ \pm 1 / 2}(\mathbb{R})$, equipped with the norm induced by $H_{+}^{ \pm 1 / 2}$ (which carries the norm of $H^{ \pm 1 / 2}$ as a closed subspace). See [20, 22] for properties of the spaces $\widetilde{H}^{s}(\Omega)$ in general.

Now we assume the following situation in 1.1 :

$$
\begin{aligned}
T & =r_{+} A_{\Phi}: H^{r, c}(\mathbb{R}) \rightarrow H^{s}\left(\mathbb{R}_{+}\right) \\
\Upsilon_{0} & =\lambda_{-}^{s} \Phi \Psi^{-1} \lambda^{q-r} \in \mathcal{G} C^{\mu}(\ddot{\mathbb{R}}) \\
\omega & =\frac{1}{2 \pi i} \int_{\mathbb{R}} d \log \Upsilon_{0}=\kappa+\eta+i \tau \\
\kappa & \in \mathbb{Z}, \tau \in \mathbb{R} \\
\eta & =\left\{\begin{array}{r}
-\frac{1}{4} \text { for } \ell^{c}=\ell^{e} \\
-\frac{3}{4} \text { for } \ell^{c}=\ell^{o} .
\end{array}\right.
\end{aligned}
$$

Proposition 5.4. If (5.6) is satisfied, then $T$ is not normally solvable but

$$
\begin{aligned}
& \alpha(T)=\operatorname{dim} \operatorname{ker} T=\max \{0,-\kappa\}<\infty \\
& \bar{\beta}(T)=\operatorname{dim} H^{s}\left(\mathbb{R}_{+}\right) / \operatorname{clos} \operatorname{im} T=\max \{0, \kappa\}<\infty .
\end{aligned}
$$

Proof. We know from Theorem 2.2 that $T \sim U_{0}$. Hence let us first normalize $U_{0}$ with the method of 10 which runs as follows. Consider the shifted operators as defined in 2.9 :

$$
U_{\delta}=r_{+} A_{\Upsilon_{0}} \ell^{c}: H^{\delta}\left(\mathbb{R}_{+}\right) \rightarrow H^{\delta}\left(\mathbb{R}_{+}\right)
$$

for $\delta \in]-1 / 2,1 / 2[$ as a restriction $(\delta>0)$ or continuous extension $(\delta<0)$ of $U_{0}$. The lifted shifted operators $U_{\delta, 0}$ of $U_{\delta}$ due to Theorem 2.2 are Fredholm: they satisfy (5.1) up to the last condition where we have

$$
\eta_{\delta, 0}=\left\{\begin{array}{lll}
\delta-\frac{1}{4} & \text { for } \quad \delta>0, & \ell^{c}=\ell^{e} \\
\delta-\frac{3}{4} & \text { for } \quad \delta>0, & \ell^{c}=\ell^{o} \\
\delta+\frac{3}{4} & \text { for } \quad \delta<0, & \ell^{c}=\ell^{e} \\
\delta+\frac{1}{4} & \text { for } \quad \delta<0, & \ell^{c}=\ell^{o} .
\end{array}\right.
$$


Further $\kappa_{\delta, 0}=\kappa$ for $\delta>0$ and $\kappa_{\delta, 0}=\kappa+1$ for $\delta<0$. A monotony argument yields, for $\delta \in] 0,1 / 2[$,

$$
\begin{aligned}
& \operatorname{ker} U_{\delta} \subset \operatorname{ker} U \subset \operatorname{ker} U_{-\delta} \\
& \operatorname{im} U_{\delta} \subset \operatorname{im} U \subset \operatorname{im} U_{-\delta} \\
& -\kappa_{\delta, 0}=\operatorname{ind} U_{\delta}=\operatorname{ind} U=-\kappa=\operatorname{ind} U_{-\delta}-1=-\kappa_{-\delta, 0}+1 .
\end{aligned}
$$

With the help of Theorem 2.2 we obtain (5.7): It is clear that the equivalence relation $T \sim U_{0}$ transfers all the mentioned properties, because it implies isomorphic kernels and co-kernels as well as isomorphic images and quotients $\operatorname{dom} T / \operatorname{ker} T \cong$ $\operatorname{dom} U_{0} / \operatorname{ker} U_{0}$.

Theorem 5.5. Under the assumptions (5.1) an image normalization of $T$ is given by

$$
\stackrel{\prec}{T}=\operatorname{Rst} T \quad: \quad H^{r, c}(\mathbb{R}) \rightarrow \stackrel{\prec}{H^{s+i \tau}}\left(\mathbb{R}_{+}\right) .
$$

The formulas (5.7) extend to

$$
\begin{aligned}
& \alpha(\stackrel{\prec}{T})=\alpha(T)=\max \{0,-\kappa\} \\
& \beta(\stackrel{\prec}{T})=\bar{\beta}(T)=\max \{0, \kappa\},
\end{aligned}
$$

i.e., $\stackrel{\prec}{T}$ is a Fredholm operator. A generalized inverse of $\stackrel{\prec}{T}$ is obtained by continuous extension of a generalized inverse of $T_{\delta}: H^{r+\delta, c}(\mathbb{R}) \rightarrow H^{s+\delta}\left(\mathbb{R}_{+}\right)$for $\left.\delta \in\right] 0,1 / 2[$, given by the formulas

$$
\begin{aligned}
& \stackrel{\prec}{T^{-}}=\underset{\operatorname{Ext} T_{\delta}^{-}}{ } \quad: \stackrel{\prec}{H^{s}}\left(\mathbb{R}_{+}\right) \rightarrow H^{r, c}(\mathbb{R}) \\
& T_{\delta}^{-}=T_{\delta, 0}^{-} \quad: \quad H^{s+\delta}\left(\mathbb{R}_{+}\right) \rightarrow H^{r+\delta, c}(\mathbb{R}) .
\end{aligned}
$$

Herein $T_{\delta, 0}^{-}$is given by Theorem 3.3 (substituting there $T^{-}$by $T_{\delta, 0}^{-}$).

Proof. Again the result is obtained by reduction to $U_{0}$, normalization of $U_{0}$ and by the fact that the equivalence relation transfers the properties in question and generates corresponding formulas. See details in the previous proof.

\section{Regularity properties of solutions}

In this section we consider the operator $T$ as given by $(1.1)$ in a scale of Bessel potential spaces, i.e., the shifted operators $T^{(\delta)}$ given by (2.11) accompanying $T$. If there is an AGF of $\Upsilon=\Phi \Psi^{-1}$, we may apply Theorem 3.3 to $T^{(\delta)}$ and obtain an immediate result, remarkably for even values of $\delta$ : 
Theorem 6.1. Let $T$ be given by (1.1) and $T^{(\delta)}$ defined by (2.11). Further let $\Upsilon=\Phi \Psi^{-1}$ admit an AGF with factorization index $\kappa \in \mathbb{Z}$. Then $T^{(\delta)}$ is Fredholm for all $\delta \in 2 \mathbb{Z}$ with analytical index ind $T^{(\delta)}=-\kappa-\delta / 2$. A generalized inverse of $T^{(\delta)}$ is given by formula 3.9 where the factorization of $\Upsilon$ is replaced by:

$$
\Upsilon=\left(\lambda_{-}^{-\delta} \Upsilon_{-}\right) \zeta^{\kappa+\delta / 2}\left(\Upsilon_{e} \lambda^{\delta}\right) \text {. }
$$

Proof. If $\Upsilon=\Upsilon_{-} \zeta^{\kappa} \Upsilon_{e}$ is an AGF of $\Upsilon=\Phi \Psi^{-1}$ with respect to $P_{1}=P^{r, e / o}$ and $P_{2}=P_{2}^{(s)}$, then 6.1 represents an AGF of $\Upsilon$ with respect to $P_{1}=P^{r+\delta, e / o}$ and $P_{2}=P_{2}^{(s+\delta)}$ provided $\delta \in 2 \mathbb{Z}$. This is because $\zeta^{\delta / 2}=\lambda_{-}^{\delta} \lambda^{-\delta}$, the new factors belong to the corresponding weighted $L^{2}$ spaces $(3.2)$ in Definition 3.1 and the composed operator corresponding to 3.3 is bounded. The last conclusion is a consequence of the lifting method. Therefore we can apply Theorem 3.3 replacing $r$ by $r+\delta$ and $s$ by $s+\delta$.

Corollary 6.2. As before let $T$ be given by 1.1 , $T^{(\delta)}$ defined by (2.11) and let $\Upsilon=\Phi \Psi^{-1}$ admit an $A G F$ with factorization index $\kappa \in \mathbb{Z}$.

I. Then the shifted operator $T^{(\delta)}$ is invertible for $\delta=-2 \kappa$, left invertible for $\delta=m-2 \kappa, m \in \mathbb{N}$ and right invertible for $\delta=m-2 \kappa, m \in-\mathbb{N}$. In these cases ind $T^{(\delta)}=-m$.

II. If $\Upsilon_{0}=\lambda_{-}^{s} \Phi \Psi^{-1} \lambda^{q-r} \in \mathcal{G} C^{\mu}(\ddot{\mathbb{R}})$, then $T^{(\delta)}$ is invertible for orders $\delta \in$ ]$-2 \kappa-3 / 2,-2 \kappa+1 / 2[$ in the e-case and for $\delta \in]-2 \kappa-1 / 2,-2 \kappa+3 / 2[$ in the o-case, respectively. $T^{(\delta)}$ is left invertible for $\left.\delta \in\right] m-2 \kappa-3 / 2, m-2 \kappa+1 / 2[$, $m \in \mathbb{N}$ in the e-case and for $\delta \in] m-2 \kappa-1 / 2, m-2 \kappa+3 / 2[, m \in \mathbb{N}$ in the $o$ case, respectively. $T^{(\delta)}$ is right invertible for $\left.\delta \in\right] m-2 \kappa-3 / 2, m-2 \kappa+1 / 2[$, $m \in-\mathbb{N}$ in the e-case and for $\delta \in] m-2 \kappa-1 / 2, m-2 \kappa+3 / 2[, m \in-\mathbb{N}$ in the o-case, respectively. Otherwise $T^{(\delta)}$ is not normally solvable.

Definition 6.3. Let $T$ be given by (1.1), $g \in H^{s}\left(\mathbb{R}_{+}\right)$and $\delta>0$. Then a solution $f \in H^{r, c}(\mathbb{R})$ of the equation

$$
T f=g
$$

is said to be $(r+\delta)$-regular if $f \in H^{r+\delta}(\mathbb{R})$.

Proposition 6.4. Let $T$ be given by 1.1), $\delta>0$ and $g \in H^{s+\delta}\left(\mathbb{R}_{+}\right)$. Further let $T^{(\delta)-}$ be a generalized inverse of $T^{(\delta)}$ (as given in Theorem 6.1, e.g.). Then a solution $f \in H^{r, c}(\mathbb{R})$ of the equation $\sqrt{6.2}$ is $(r+\delta)$-regular if and only if

$$
T T^{(\delta)-} g=g \text {. }
$$

Proof. By assumption $T^{(\delta)} T^{(\delta)-} T^{(\delta)}=T^{(\delta)}$, i.e., $T^{(\delta)} T^{(\delta)-}$ is a projector onto $\operatorname{im} T^{(\delta)}$. If $f \in H^{r, c}(\mathbb{R})$ is a solution of the equation 6.2 in $H^{r+\delta}(\mathbb{R})$, it is a solution of $T^{(\delta)} f=g$ and therefore $T^{(\delta)} T^{(\delta)-} g=g$ which can be also written as (6.3). The inverse conclusion holds obviously, as well.

Under certain conditions, the projector $T^{(\delta)} T^{(\delta)-}$ can be formulated in terms of the factors of an AGF of $\Upsilon$ as follows: 
Proposition 6.5. Let $T$ be given by (1.1), $\delta \in 2 \mathbb{Z}, \delta>0$ and $g \in H^{s+\delta}\left(\mathbb{R}_{+}\right)$. Further let $\Upsilon=\Upsilon_{-} \zeta^{\kappa} \Upsilon_{e}$ be an $A G F$ with respect to $\left(L^{2}\left(\mathbb{R}, \lambda^{r}\right), L^{2}\left(\mathbb{R}, \lambda^{s}\right), \Pi_{1}, \Pi_{2}\right)$ (see Definition 3.1). Moreover let $\delta \geq-2 \kappa$ (such that $T^{(\delta)}$ is left invertible, see Theorem 3.3). Then a solution $f \in H^{r, c}(\mathbb{R})$ of the equation 6.2 is $(r+\delta)$-regular if and only if

$$
r_{+} A_{\phi^{-1}} \ell^{c} r_{+} A_{\phi} \ell g=g
$$

where $\ell g$ is any extension of $g$ in $H^{s+\delta}(\mathbb{R})$ and

$$
\phi=\Upsilon_{-}^{-1} \zeta^{-\kappa} \lambda^{\delta} .
$$

Proof. We simplify the projector in 6.3):

$$
\begin{aligned}
T^{(\delta)} T^{(\delta)-}= & \left.r_{+} A_{\Phi \Psi^{-1}} P^{e / o} A_{\Psi}\right|_{H^{r+\delta}} \\
& \left.A_{\Psi}^{-1} P^{e / o} \Lambda^{-\delta} A_{e}^{-1} \ell^{c} r_{+} C^{-1} A_{\zeta^{-\delta / 2}} \ell^{c} r_{+} A_{-}^{-1} \Lambda_{-}^{\delta}\right|_{\mathrm{im} P_{2}^{(s+\delta)}} \\
= & \left.r_{+} A_{-} A_{\zeta^{\kappa}} A_{e} P^{e / o} A_{\lambda^{-\delta}} A_{e}^{-1} \ell^{c} r_{+} A_{\zeta^{-\kappa-\delta / 2}} \ell^{c} r_{+} A_{-}^{-1} A_{\lambda_{-} \delta}\right|_{\mathrm{im} P_{2}^{(s+\delta)}} \\
= & \left.r_{+} A_{-} A_{\zeta^{\kappa}} A_{\lambda^{-\delta}} \ell^{c} r_{+} A_{\zeta^{-\kappa-\delta / 2}} \ell^{c} r_{+} A_{-}^{-1} A_{\lambda_{-}^{\delta}}\right|_{\operatorname{im} P_{2}^{(s+\delta)}} \\
= & \left.r_{+} A_{-} A_{\zeta^{\kappa}} A_{\lambda^{-\delta}} \ell^{c} r_{+} A_{\zeta^{-\kappa-\delta / 2}} A_{-}^{-1} A_{\lambda_{-}^{\delta}}\right|_{\operatorname{im} P_{2}^{(s+\delta)}} .
\end{aligned}
$$

This coincides with the projector in 6.4 because $\phi$ is a "minus factor", i.e. $\left.r_{+} A_{\phi}\right|_{H_{-}^{(s+\delta)}}=0$ or $r_{+} A_{\phi}=r_{+} A_{\phi} \ell r_{+}$for any extension $\ell$.

Similarly as in Corollary 6.2 one can extend the previous results to further orders $\delta \in \mathbb{R}$ (instead of only $\delta \in 2 \mathbb{Z}$ ) if the symbols are Hölder continuous.

Corollary 6.6. Let $T$ be given by (1.1), $\Upsilon_{0}=\lambda_{-}^{s} \Phi \Psi^{-1} \lambda^{q-r} \in \mathcal{G} C^{\mu}(\ddot{\mathbb{R}})$ and $\omega=$ $\frac{1}{2 \pi i} \int_{\mathbb{R}} d \log \Upsilon_{0}$ (cf. Theorem 4.3). Moreover let $T^{(\delta)}$ be defined by (2.11), and assume $\delta>0, \Re e(\omega)+\delta \pm \frac{1}{4} \notin \mathbb{Z}$ in the e/o-case, respectively.

Then, a solution $f$ of the equation $(6.2)$ is $r+\delta$-regular if and only if $(6.3)$ is fulfilled. Under the conditions of Corollary 6.2, part II, it gains the form of (6.4).

Remark 6.7. In the exceptional cases where $T^{(\delta)}$ is not normally solvable, we obtain regularity results after normalization by the help of Section 5 .

\section{Applications}

A class of canonical problems of diffraction theory 24 leads to the study of the following very basic BVPs for the Helmholtz equation in a quadrant, briefly written as

$$
\begin{aligned}
& A u(x)=\left(\Delta+k^{2}\right) u(x)=\left(\frac{\partial^{2}}{\partial x_{1}^{2}}+\frac{\partial^{2}}{\partial x_{2}^{2}}+k^{2}\right) u(x)=0 \quad \text { in } \quad Q_{1} \\
& B_{1} u(x)=\left(\alpha u+\beta \frac{\partial u}{\partial x_{2}}+\gamma \frac{\partial u}{\partial x_{1}}\right)(x)=g_{1}(x) \quad \text { on } \quad \Gamma_{1} \\
& B_{2} u(x)=\left(\alpha^{\prime} u+\beta^{\prime} \frac{\partial u}{\partial x_{1}}+\gamma^{\prime} \frac{\partial u}{\partial x_{2}}\right)(x)=g_{2}(x) \quad \text { on } \quad \Gamma_{2},
\end{aligned}
$$


where e.g. $Q_{1}$ denotes the first (open) quadrant in $\mathbb{R}^{2}$ bordered by the coordinate semi-axes $\Gamma_{1}=\left\{x=\left(x_{1}, x_{2}\right) \in \mathbb{R}^{2}: x_{1} \geq 0, x_{2}=0\right\}$ and $\Gamma_{2}=\left\{x=\left(x_{1}, x_{2}\right) \in\right.$ $\left.\mathbb{R}^{2}: x_{1}=0, x_{2} \geq 0\right\} ;$ cf. Figure 1 .

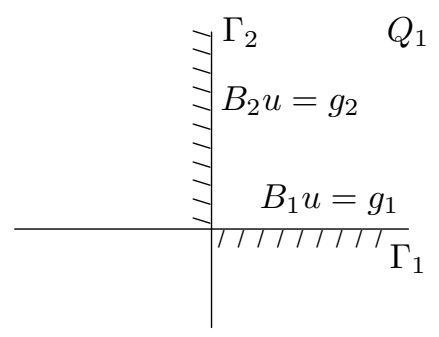

Figure 1. The geometry of the BVPs for the Helmholtz equation in a quadrant.

These have been extensively investigated and partly solved in weak formulation, i.e., looking for $u \in H^{1}\left(Q_{1}\right)$ for any given $g_{j} \in H^{s_{j}}\left(\Gamma_{j}\right), s_{j}= \pm 1 / 2(j=1,2)$ 10, 11 provided the boundary operators are of "normal type", i.e., certain Fourier symbols do not degenerate (where the case of $s_{j}=+1 / 2$ corresponds with a Dirichlet condition).

Also questions concerning small regularity (i.e., $u \in H^{1+\varepsilon}\left(Q_{1}\right), \varepsilon \in[0,1 / 2[$ ) were answered, but not yet studied for higher values of $\varepsilon$. Those investigations were based upon the generalized inversion of certain operator matrices of the form

$$
\mathcal{T}=\left(\begin{array}{cc}
T_{1} & K_{1} \\
K_{2} & T_{2}
\end{array}\right)
$$

where $T_{1}, T_{2}$ are CTOS (or Wiener-Hopf plus/minus Hankel operators) of the present form acting in trace spaces $H^{ \pm 1 / 2+\varepsilon}\left(\mathbb{R}_{+}\right)$and $K_{1}, K_{2}$ are certain Fourier integral operators, causing some complications.

Here we present a new approach for the explicit solution of the BVPs (7.1), valid also for more general problems, that is based upon symmetry which yields equivalent reduction to scalar CTOS and includes normalization and regularity results. Moreover the method is simpler and more transparent. The case of Dirichlet conditions will be omitted for simplicity, however can be tackled analogously.

Definition 7.1. Let $\mathcal{H}^{1}(\Omega)$ denote the space of weak solutions of the Helmholtz equation in a domain $\Omega \subset \mathbb{R}^{2}$ such as the first quadrant $Q_{1}$ or the upper halfplane $Q_{12}$ equipped with the $H^{1}$-norm. Further let $\mathcal{H}^{1+\varepsilon}(\Omega)=\mathcal{H}^{1}(\Omega) \cap H^{1+\varepsilon}(\Omega)$. Then the operator

$$
L=\left(B_{1}, B_{2}\right)^{T}: \mathcal{H}^{1}\left(Q_{1}\right) \rightarrow H^{-1 / 2}\left(\Gamma_{1}\right) \oplus H^{-1 / 2}\left(\Gamma_{2}\right)
$$

is referred to as the operator associated with the BVP 7.1$). L^{(\varepsilon)}$ stands for the shifted operator (cf. Section 2, formula (2.11) ) in the spaces of smoother functions: $L^{(\varepsilon)}: \mathcal{H}^{1+\varepsilon}\left(Q_{1}\right) \rightarrow H^{-1 / 2+\varepsilon}\left(\Gamma_{1}\right) \oplus H^{-1 / 2+\varepsilon}\left(\Gamma_{2}\right), \varepsilon>0$. 
Moreover, $L_{0}$ denotes the operator associated with the semi-homogeneous $B V P$ (7.1) where $g_{2}=0$, i.e.,

$$
L_{0}=\operatorname{Rst} B_{1}:\left\{u \in \mathcal{H}^{1}\left(Q_{1}\right): B_{2} u=0\right\} \rightarrow H^{-1 / 2}\left(\Gamma_{1}\right) .
$$

$L_{0}^{(\varepsilon)}$ is defined analogously. From now on the half-lines $\Gamma_{j}$ will be identified with $\mathbb{R}_{+}$, if their is no risk of misunderstanding.

It is clear that the BVPs are well-posed, if and only if the corresponding associated operators are boundedly invertible.

Note that (in the present case of $s_{j}=-1 / 2$ or $\left.s_{j}+\varepsilon \in\right]-1 / 2,1 / 2[$ ) the boundary operators can be understood as $B_{j}=r_{+} A_{\sigma_{j}} \ell_{0} T_{0, \Gamma_{j}}$, where the Fourier symbols of $A_{\sigma_{j}}$ (also called pre-symbols of $B_{j}$ ) play a crucial role:

$$
\sigma_{1}=\alpha-\beta t+\gamma \vartheta \quad, \quad \sigma_{2}=\alpha^{\prime}-\beta^{\prime} t+\gamma^{\prime} \vartheta,
$$

with $t(\xi)=\xi^{2}-k^{2}, \vartheta(\xi)=i \xi, \xi \in \mathbb{R}$ (and the identification of $\Gamma_{j}$ with $\mathbb{R}_{+}$), see [10. Further we shall need the companion operators $B_{j}^{*}$ of $B_{j}$ with pre-symbols

$$
\sigma_{1 *}=\alpha-\gamma t+\beta \vartheta \quad, \quad \sigma_{2 *}=\alpha^{\prime}-\gamma^{\prime} t+\beta^{\prime} \vartheta
$$

i.e., where the role of the two variables is exchanged (in comparison with $\sigma_{1}$ and $\sigma_{2}$, respectively).

The consideration of a "lifted operator" of $L$ (in the sense of Theorem 2.2) seems not convenient in the present situation, since the representation of Bessel potential operators for the quarter-plane is rather complicated, see [14, 15].

Definition 7.2. Two operators acting in Banach spaces are called equivalent after extension (EAE), in brief $S * T$, 2, if there are Banach spaces $Z_{1}, Z_{2}$ and linear homeomorphisms $E, F$ such that

$$
\left(\begin{array}{cc}
S & 0 \\
0 & I_{Z_{1}}
\end{array}\right)=E\left(\begin{array}{cc}
T & 0 \\
0 & I_{Z_{2}}
\end{array}\right) F
$$

Properties of this kind of relationship are described in [2, 8, 21, 38]. In the present context it is most important that an EAE relationship implies that the two operators have isomorphic kernels and isomorphic co-kernels, hence are only simultaneously Fredholm, one-sided invertible etc. A formula for a generalized inverse of $S$ or $T$ implies a formula for a generalized inverse of the other, provided $E, F$ or $E^{-1}, F^{-1}$, respectively, are known.

Proposition 7.3. Let $L$ and $L_{0}$ be given as before and $B_{2}: \mathcal{H}^{1}\left(Q_{1}\right) \rightarrow H^{-1 / 2}\left(\Gamma_{2}\right)$ be right invertible. Then $L \stackrel{*}{\sim} L_{0}$.

Proof. This result is known from a general operator setting, see Theorem 3.2 in [35. In the present context, if $R$ is a right inverse of $B_{2}$, i.e. $B_{2} R=\left.I\right|_{H^{-1 / 2}\left(\Gamma_{2}\right)}$, then an EAE relation between $L$ and $L_{0}$ can be written as

$$
L=\left(\begin{array}{c}
B_{1} \\
B_{2}
\end{array}\right)=\left(\begin{array}{cc}
\left.I\right|_{H^{-1 / 2}\left(\Gamma_{1}\right)} & \left.B_{1}\right|_{X_{1}} \\
0 & \left.B_{2}\right|_{X_{1}}
\end{array}\right)\left(\begin{array}{cc}
\left.B_{1}\right|_{X_{0}} & 0 \\
0 & \left.I\right|_{X_{1}}
\end{array}\right)\left(\begin{array}{c}
P \\
Q
\end{array}\right)
$$


where we put $P=I-R B_{2}, Q=R B_{2}$ which are continuous projectors in $\mathcal{H}^{1}\left(Q_{1}\right)$, $X_{0}=\operatorname{ker} B_{2}=\operatorname{im} P=\operatorname{ker} Q, X_{1}=\operatorname{im} Q=\operatorname{ker} P$.

Corollary 7.4. In this situation, if $L_{0}$ is generalized invertible by $L_{0}^{-}$, i.e.,

$$
L_{0} L_{0}^{-} L_{0}=L_{0} \text {, }
$$

then $L$ is generalized invertible, as well, and a generalized inverse of $L$ follows from (7.8) by the reverse order law (cf. 35], Theorem 4.5):

or

$$
L^{-}=(P, Q)\left(\begin{array}{cc}
L_{0}^{-} & 0 \\
0 & \left.I\right|_{X_{1}}
\end{array}\right)\left(\begin{array}{cc}
\left.I\right|_{H^{-1 / 2}\left(\Gamma_{1}\right)} & -\left.B_{1}\right|_{X_{1}} R \\
0 & R
\end{array}\right)
$$

$L^{-}\left(\begin{array}{l}g_{1} \\ g_{2}\end{array}\right)=L_{0}^{-}\left(g_{1}-B_{1} R g_{2}\right)+R g_{2} \quad, \quad\left(g_{1}, g_{2}\right) \in H^{-1 / 2+\varepsilon}\left(\Gamma_{1}\right) \oplus H^{-1 / 2+\varepsilon}\left(\Gamma_{2}\right)$.

Surely, if $L_{0}$ is invertible, then $L_{0}^{-}$coincides with $L_{0}^{-1}$, and the formula represents the resolvent operator (inverse of (7.3)).

Proposition 7.5. Let $B_{2}$ be given as in (7.1) and its Fourier symbol $\sigma_{2}=\alpha^{\prime}-\beta^{\prime} t+$ $\gamma^{\prime} \vartheta$ be invertible of order 1 . Then the following holds:

I. The boundary operator $B_{2}^{(\varepsilon)}: \mathcal{H}^{1+\varepsilon}\left(Q_{1}\right) \rightarrow H^{\varepsilon-1 / 2}\left(\mathbb{R}_{+}\right)$is right invertible for all $\varepsilon \geq 0$.

II. $L^{(\varepsilon)} \stackrel{*}{\sim} L_{0}^{(\varepsilon)}$ for all $\varepsilon \geq 0$.

Proof. I. It is well-known that, for every $s>0$, there exists a continuous extension operator $\ell^{s}: H^{s}\left(\mathbb{R}_{+}\right) \rightarrow H^{s}(\mathbb{R})$ which is left invertible by restriction $r_{+}$, for instance $\Lambda_{-}^{-s} \ell_{0} r_{+} \Lambda_{-}^{s} \ell$ where $\ell \varphi$ is any extension of $\varphi \in H^{s}\left(\mathbb{R}_{+}\right)$in $H^{s}(\mathbb{R})$ (which exists by definition of the space $\left.H^{s}\left(\mathbb{R}_{+}\right)\right)$. Now consider

$$
R^{\varepsilon} \varphi\left(x_{1}, x_{2}\right)=\mathcal{F}_{\xi \mapsto x_{2}}^{-1} e^{-t(\xi) x_{1}} \sigma_{2}^{-1}(\xi) \widehat{\ell^{\varepsilon-1 / 2}} \varphi(\xi), \quad\left(x_{1}, x_{2}\right) \in Q_{1} .
$$

It is easy to verify that $R^{\varepsilon}$ is a bounded linear operator, acting from $H^{\varepsilon-1 / 2}\left(\mathbb{R}_{+}\right)$ into $\mathcal{H}^{1+\varepsilon}\left(Q_{1}\right)$ and, moreover, that $B_{2} R^{\varepsilon}=\left.I\right|_{H^{\varepsilon-1 / 2}\left(\mathbb{R}_{+}\right)}$. Since $B_{2}^{(\varepsilon)}$ is a bounded linear operator, we obtain the first result.

II. The operator matrix identity $(7.8)$ holds for the shifted operators, as well. This implies the second statement.

Definition 7.6. The following problem is referred to as a BVP with symmetry for the Helmholtz equation in the upper half-plane of $\mathbb{R}^{2}$ (see Figure 2):

$$
\begin{aligned}
& A u(x)=\left(\Delta+k^{2}\right) u(x)=\left(\frac{\partial^{2}}{\partial x_{1}^{2}}+\frac{\partial^{2}}{\partial x_{2}^{2}}+k^{2}\right) u(x)=0 \quad \text { in } \quad Q_{12} \\
& B_{1} u(x)=\left(\alpha u+\beta \frac{\partial u}{\partial x_{2}}+\gamma \frac{\partial u}{\partial x_{1}}\right)(x)=g_{1}(x) \quad \text { on } \Gamma_{1} \\
& B_{2} u(x)=\left(\alpha^{\prime} u+\beta^{\prime} \frac{\partial u}{\partial x_{1}}+\gamma^{\prime} \frac{\partial u}{\partial x_{2}}\right)(x)=0 \quad \text { on } \quad \Gamma_{2} .
\end{aligned}
$$

Here we are looking for $u \in H^{1}\left(Q_{12}\right)$ for any given $g_{1} \in H^{-1 / 2}\left(\Gamma_{1}\right)$. 


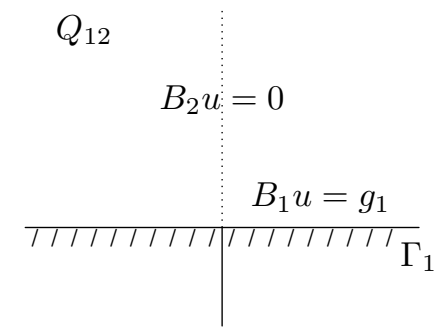

FiguRE 2. The geometry of the BVP with symmetry for the Helmholtz equation in the upper half-plane.

The name "BVP with symmetry" is motivated by the subsequent results:

Lemma 7.7. Let $u \in \mathcal{H}^{1}\left(Q_{12}\right), B_{2}$ be defined as before and $A_{\sigma_{2 *}}$ be invertible of order 1 . Then the following statements are equivalent:

(i) $B_{2} u=0$,

(ii) $A_{\sigma_{2 *}}^{-1} P^{e} A_{\sigma_{2 *}} u_{0}=0$ where $u_{0}$ is the trace of $u$ on the boundary line $x_{2}=0$,

(iii) $A_{\sigma_{2 *}}^{-1} P^{e} A_{\sigma_{2 *}} u_{0, a}=0, a \geq 0$ where $u_{0, a}$ is the trace of $u$ on any line $x_{2}=a$.

Proof. We know that the Dirichlet problem for the Helmholtz equation in the upper half-plane is well-posed and explicitly solved by the formula

$$
u\left(x_{1}, x_{2}\right)=\mathcal{K}_{D, Q_{12}} u_{0}=\mathcal{F}_{\xi \mapsto x_{1}}^{-1} e^{-t(\xi) x_{2}} \widehat{u_{0}}(\xi), \quad\left(x_{1}, x_{2}\right) \in Q_{12}
$$

provided $u_{0}=g \in H^{1 / 2}(\mathbb{R})$ is given on $x_{2}=0$, see [26, 33], for instance. Now condition (i) implies:

$$
\begin{gathered}
B_{2} u\left(0, x_{2}\right)=\left.\mathcal{F}_{\xi \mapsto x_{1}}^{-1} \sigma_{2 *}(\xi) e^{-t(\xi) x_{2}} \widehat{u_{0}}(\xi)\right|_{x_{1}=0}=0, x_{2} \in \mathbb{R}_{+} \\
\left.\frac{1}{\sqrt{2 \pi}} \int_{-\infty}^{+\infty} e^{-i \xi x_{1}-t(\xi) x_{2}} \widehat{A_{\sigma_{2 *}} u_{0}}(\xi) d \xi\right|_{x_{1}=0}=0, x_{2} \in \mathbb{R}_{+} \\
A_{-t x_{2}} A_{\sigma_{2 *}} \widehat{u_{0}}(\xi)=0, x_{2} \in \mathbb{R}_{+} .
\end{gathered}
$$

Since $t$ is an even function, this is possible only for an odd functional $\widehat{A_{\sigma_{2 *}} u_{0}}$ and therefore only if $A_{\sigma_{2 *}} u_{0}$ is odd (cf. [11, Lemma 3.4), i.e., (ii) is satisfied.

The inverse conclusion ((ii) yields (i)) is obvious from the same calculation.

Hence, a representation formula for $u \in \mathcal{H}^{1}\left(Q_{12}\right)$ satisfying $B_{2} u=0$ on $\Gamma_{2}$ is given by

$$
\begin{aligned}
u\left(x_{1}, x_{2}\right) & =\mathcal{K}_{B_{2 *}, Q_{12}} h=\mathcal{F}_{\xi \mapsto x_{1}}^{-1} e^{-t(\xi) x_{2}} \widehat{S^{-1} h}(\xi), \quad\left(x_{1}, x_{2}\right) \in Q_{12} \\
S & =A_{\sigma_{2 *}}, \quad h \in H^{-1 / 2, o} .
\end{aligned}
$$

This implies (iii) and the inverse conclusion holds by similar arguments as before. 
Corollary 7.8. A weak solution of the Helmholtz equation in $Q_{12}$ satisfies the condition $B_{2} u=0$ in 7.10 if and only if

$$
S^{-1} P^{o} S u\left(\cdot, x_{2}\right)=u\left(\cdot, x_{2}\right), \quad x_{2} \in[0, \infty[
$$

where $S=A_{\sigma_{2 *}}$.

Let us write this subspace as $\mathcal{H}^{1, c}\left(Q_{12}\right)=\left\{u \in \mathcal{H}^{1}\left(Q_{12}\right): S^{-1} P^{e} S u=0\right\}$. The operator associated with 7.10 is referred to as $L_{0, c}$, the shifted operators by

$$
L_{0, c}^{(\varepsilon)}: \quad \mathcal{H}^{1+\varepsilon, c}\left(Q_{12}\right) \rightarrow H^{\varepsilon-1 / 2}\left(\mathbb{R}_{+}\right), \quad \varepsilon \geq 0 .
$$

Theorem 7.9. Let $L, L_{0}, L_{0, c}$ be the operators associated with the BVPs defined before (see (7.3), (7.4), (7.14) where $A_{\sigma_{2 *}}$ is invertible of order 1 , and let $L^{(\varepsilon)}$, $L_{0}^{(\varepsilon)}, L_{0, c}^{(\varepsilon)}$ denote the shifted operators for $\varepsilon>0$. Then we have

$$
\begin{aligned}
& L \stackrel{*}{\sim} L_{0} \sim L_{0, c} \sim T=r_{+} A_{\Upsilon}: H^{-1 / 2, o} \rightarrow H^{-1 / 2}\left(\mathbb{R}_{+}\right), \\
& L^{(\varepsilon)} \stackrel{*}{\sim} L_{0}^{(\varepsilon)} \sim L_{0, c}^{(\varepsilon)} \sim T^{(\varepsilon)}=\operatorname{Rst} r_{+} A_{\Upsilon}: H^{\varepsilon-1 / 2, o} \rightarrow H^{\varepsilon-1 / 2}\left(\mathbb{R}_{+}\right)
\end{aligned}
$$

where $\Upsilon=\sigma_{1} \sigma_{2 *}^{-1}$ and all relations are explicitly computed from the preceding formulas.

Proof. The first relation in both lines of 7.15 results from a combination of the statements of Proposition 7.3 and Proposition 7.4. Furthermore Corollary 7.7 implies that there is a projector onto $\mathcal{H}^{1, c}\left(Q_{12}\right)$ :

$$
\Pi=S^{-1} P^{o} S: \mathcal{H}^{1}\left(Q_{12}\right) \rightarrow \mathcal{H}^{1, c}\left(Q_{12}\right) .
$$

Therefore

$$
L_{0} r_{Q_{1}}=L_{0, c} \quad, \quad L_{0}=L_{0, c} \Pi \ell
$$

where $r_{Q_{1}}$ denotes the restriction of $u \in \mathcal{H}^{1, c}\left(Q_{12}\right)$ to $\operatorname{dom} L_{0}$ (see 7.4$)$ ) and $\ell$ denotes any extension from $\operatorname{dom} L_{0}$ to $\mathcal{H}^{1}\left(Q_{12}\right)$. This yields the second relation in the first line of 7.15 and in the second line, as well, by restriction.

The third relation is finally a consequence of (7.9), (7.11), both admitting parameters $\varepsilon \geq 0$, which yield:

$$
T S T_{0, \partial Q_{12}}=L_{0, c} \quad, \quad T=L_{0, c} \mathcal{K}_{D, Q_{12}} S^{-1}
$$

where $T_{0, \partial Q_{12}}$ is the trace operator acting into $H^{1 / 2}\left(\partial Q_{12}\right), \partial Q_{12}=\left\{\left(x_{1}, x_{2}\right) \in\right.$ $\left.\mathbb{R}^{2}: x_{2}=0\right\}$.

Theorem 7.10. Let the assumptions of Theorem 7.9 be satisfied, moreover let $\Upsilon_{0}=$ $\lambda_{-}^{-1 / 2} \Upsilon \lambda^{1 / 2} \in \mathcal{G} C^{\mu}(\ddot{\mathbb{R}})$ and put $\omega=\frac{1}{2 \pi i} \int_{\mathbb{R}} d \log \Upsilon_{0}$. Then the following holds:

I. $L$ is invertible (the BVP (7.1) is well-posed) if and only if $\Re e(\omega) \in]-\frac{3}{4}, \frac{1}{4}[$.

II. $L$ is Fredholm if and only if $\Re e(\omega)-\frac{1}{4} \notin \mathbb{Z}$.

III. The following conditions are equivalent:

(i) $L$ is normally solvable,

(ii) $L$ is generalized invertible,

(iii) $L$ is one-sided invertible, 
(iv) L is Fredholm (cf. II).

$I V$. In the last case, an explicit formula for a generalized inverse $L^{-}$of $L$ is given by (3.9) and the formulas behind (7.15) representing in case I the so-called resolvent operator of the BVP: $u=L^{-1}\left(g_{1}, g_{2}\right)$.

$V$. If $\Re e(\omega)-\frac{1}{4} \in \mathbb{Z}, L$ can be normalized by minimal image normalization in the sense of Definition 5.1 by Theorem 5.5 and the relations of Theorem 7.8 .

Proof. All statements follow from the previous results, by recalling the corresponding properties of $T$ with the help of Section 4 and transferring the corresponding properties from $T$ to $L$ with the help of Theorem 7.9.

Corollary 7.11. Regularity results for the solution of the foregoing BVPs in the scale of Bessel potential spaces are obtained from Section 5 considering $L^{(\varepsilon)}$ and the operators in the second line of 7.15 with analogous conclusions as in the case of $\varepsilon=0$ before.

Remark 7.12. Looking at Theorem 7.9 we find an alternative way to the solution, provided $A_{\sigma_{1 *}}$ is invertible of order 1 (instead of or additional to $A_{\sigma_{2 *}}$ being invertible of order 1 ), namely if

$$
\Upsilon_{*}^{-1}=\left(\sigma_{1} \sigma_{2 *}^{-1}\right)_{*}^{-1}=\sigma_{2} \sigma_{1 *}^{-1}
$$

admits an AGF alternatively or additionally. In the last case, we have the following:

Proposition 7.13. Consider the BVP (7.1) where both $\sigma_{j}$ and both $\sigma_{j *}$ are invertible of order 1. Then the problem is well-posed (i.e., $L$ is boundedly invertible), if and only if the two symbols $\Upsilon=\sigma_{1} \sigma_{2 *}^{-1}$ and $\Upsilon_{*}^{-1}=\sigma_{2} \sigma_{1 *}^{-1}$ admit canonical AGFs with respect to $H^{-1 / 2}$ and $P^{o}$.

Proof. In brief, this is a consequence of Theorem 7.10 together with Theorem 3.4 of 35] which tells us, in an abstract setting (applicable to the present situation), that both semi-homogeneous problems (corresponding with $g_{1}=0$ and $g_{2}=0$, respectively) have to be well-posed.

Remark 7.14. In various basic applications the assumptions of Proposition 7.13 are not satisfied in the intuitive space setting, but normalization (in the above sense) helps to come to a well-posed problem, as shown in the subsequent examples. Regularity results are possible under certain smoothness and compatibility conditions for the data. This discussion will be continued in a future publication about the efficient solution of further canonical BVPs. Now we expose only few details to demonstrate the relevance and efficiency of the method.

Example. The two-impedance problem is characterized by 7.1 with boundary conditions

$$
\left(\frac{\partial}{\partial n}+i p_{j}\right) u=g_{j} \in H^{-1 / 2}\left(\mathbb{R}_{+}\right), j=1,2
$$


where $\frac{\partial}{\partial n}$ denotes the normal derivative (directed to the interior of $Q_{1}$ ) and the coefficients are specified as $\alpha=i p_{1}, \alpha^{\prime}=i p_{2}, \beta=\beta^{\prime}=1, \gamma=\gamma^{\prime}=0$. Hence we study the operator $T$ of 7.15 with

$$
\Upsilon(\xi)=\sigma_{1}(\xi) \sigma_{2 *}^{-1}(\xi)=\frac{i p_{1}-t(\xi)}{i p_{2}+\vartheta(\xi)}=-i \frac{t(\xi)-i p_{1}}{\xi-p_{2}}, \xi \in \mathbb{R} .
$$

The physically more relevant case is $\Im m p_{j}>0$, see [26], Section 5 and loc. cit. In this case both $\sigma_{j}$ and both $\sigma_{j *}$ are invertible of order $1, \sigma_{1}$ is even and $\sigma_{2 *}$ is "minus type". I.e., we have a factorization

$$
\begin{aligned}
A_{\Upsilon}=A_{-} A_{e} & =\quad A_{\sigma_{2 *}^{-1}} A_{\sigma_{1}} \\
& : H^{-1 / 2} \longleftarrow H^{-3 / 2} \longleftarrow H^{-1 / 2} .
\end{aligned}
$$

Now, this is not an AGF because of the order of the middle space. However, considering the shifted operator $\left.T_{0}^{(\varepsilon)}, \varepsilon \in\right] 0,1[$, it becomes a canonical AGF (or FIS as mentioned in Corollary 4.4):

$$
\begin{aligned}
& A_{\Upsilon}=A_{-} A_{e}=A_{\sigma_{2 *}^{-1}} \quad A_{\sigma_{1}} \\
& \text { : } \quad H^{\varepsilon-1 / 2} \longleftarrow H^{\varepsilon-3 / 2} \longleftarrow H^{\varepsilon-1 / 2}
\end{aligned}
$$

and $T_{0}^{(\varepsilon)}$ is boundedly invertible for these values of the parameter $\varepsilon$, directly by our factorization method.

Therefore we have all the foregoing consequences including the well-posedness of the BVP and explicit solution for these values of $\varepsilon \in] 0,1[$, in coincidence with results of [1], Section 4 (derived less directly).

Moreover we obtain regularity results as described in Section 6 , the representation 6.1 of a generalized inverse of $T^{(\delta)}$ for $\left.\delta \in\right] m-1 / 2, m+3 / 2[, m \in \mathbb{N}$ from Corollary 6.2 (o-case) and normalization results for $\delta \in 1 / 2+2 \mathbb{N}$ easily taken from Section 5 .

In the case of $\Im m p_{2}<0$, the symbol $\sigma_{2 *}$ is "plus type" and the factorization 7.19 has to be modified according to the following AGFs

$$
\begin{aligned}
\xi-p_{2} & =\left(\xi+p_{2}\right)^{-1}\left(\xi^{2}-p_{2}^{2}\right), \\
\sigma_{2 *}^{-1}=i\left(\xi-p_{2}\right)^{-1} & =i\left(\xi+p_{2}\right)\left(\xi^{2}-p_{2}^{2}\right)^{-1}, \\
\sigma_{1} \sigma_{2 *}^{-1}=\left(t-i p_{1}\right) i\left(\xi-p_{2}\right)^{-1} & =i\left(\xi+p_{2}\right) \frac{t-i p_{1}}{\xi^{2}-p_{2}^{2}} .
\end{aligned}
$$

Surprisingly or not, the same orders appear in the factors as in 7.19 and lead to the same conclusions about well-posedness of the BVP etc. as before. Comparing with [1], pp. 16-21, we found here a method that is more efficient and far-reaching (in view of regularity and normalization).

The compatibility condition on the data $g_{j}$, which is necessary to make the problem normally solvable in the case $\varepsilon=0$ can also be derived by consideration of the following example. 
Example. The Neumann problem can be regarded as a special case of the previous one with $p_{1}=p_{2}=0$. Here both symbols $\sigma_{j *}$ are not invertible of order 1 and the present method fails, at least if we try to apply it directly. On the other hand, the basic results are known [10, 25]: $L$ as defined in (7.3) is not normally solvable, its image is not closed, a compatibility condition on the data is necessary for the solution, namely

$$
g_{1}+g_{2} \in \widetilde{H}^{-1 / 2}\left(\mathbb{R}_{+}\right)
$$

which is a dense, proper subspace of $H^{-1 / 2}\left(\mathbb{R}_{+}\right)$. The operator is normalized by a corresponding change of the image space, in the sense of Section 5 . The shifted operators $L^{(\varepsilon)}$ are boundedly invertible for $\left.\varepsilon \in\right] 0,1[$ and do not need any additional condition 10. Considering higher values of $\varepsilon$ we meet the situation described in Section 5. Thus similar results as before come out by analogy using the fact that the operator associated with the BVP satisfies relations of the kind $(7.15)$ after normalization. Concrete results for $\varepsilon \geq 1$ will be described in detail in a separate paper.

The compatibility condition for the two-impedance problem in the case $\varepsilon=0$ coincides with (7.22), because the data $g_{1}, g_{2}$ of 7.17 differ from the normal derivatives only by terms in $H^{1 / 2}\left(\mathbb{R}_{+}\right)$which is a subspace of $\widetilde{H}^{-1 / 2}\left(\mathbb{R}_{+}\right)[28$.

Example. In the oblique derivatives problem we have $\alpha=\alpha^{\prime}=0,(\beta, \gamma),\left(\beta^{\prime}, \gamma^{\prime}\right) \in$ $\mathbb{R}^{2}$ indicating directions of the oblique derivatives of $u$ given on $\Gamma_{1}$ and $\Gamma_{2}$, respectively. The operator $T$ of 7.15 has a Fourier symbol

$$
\Upsilon(\xi)=\sigma_{1}(\xi) \sigma_{2 *}^{-1}(\xi)=\frac{\beta t+i \gamma \xi}{\gamma^{\prime} t+i \beta^{\prime}}, \quad \xi \in \mathbb{R}
$$

It is not difficult to find the parameters where $\Upsilon_{0}=\lambda_{-}^{-1 / 2} \Upsilon \lambda^{1 / 2} \in \mathcal{G} C^{\mu}(\ddot{\mathbb{R}})$ and to carry out the conditions of Section 4 for an AGF etc.

In brief, this class of symbols allows a more direct application of the present method with all the consequences outlined in the foregoing part. Actually, for different parameters, there appear operators $L$ which are invertible or Fredholm and one-sided invertible with index \pm 1 , or non-normally solvable, see a detailed discussion in [11, Section 6 . Here we have the possibility to obtain regularity and normalization results including the discovery of necessary compatibility conditions directly from the theoretical part. We summarize this technique as follows.

First we characterize the oblique derivative problems which are of normal type, but not normally solvable, i.e., the Fourier symbol 7.23 does not degenerate, but the image of the associated operator $L$ (analogously $L^{\varepsilon}, \varepsilon>0$ ) is not closed. So is the situation for the related operators $L_{0}, L_{0, c}$ and $T$, as well, according to Theorem 7.9. In view of Definition 7.1 and Corollary 7.4 the reduced equation (due to the semi-homogeneous problem) reads

$$
L_{0} u_{0}=g_{0}=g_{1}-B_{1} R g_{2} \text { for } u_{0} \in \mathcal{H}^{1}\left(Q_{1}\right) \text { with } B_{2} u_{0}=0
$$


where $R$ is given by 7.9 for instance. Theorem 7.9 gives us the equivalent symmetrized equation

$$
L_{0, c} v=L_{0} r_{Q_{1}} v=g_{0} \text { for } v \in \mathcal{H}^{1, c}\left(Q_{12}\right)
$$

and, moreover, an equivalent equation where the (unlifted) CTOS appears:

$$
T w=L_{0, c} \mathcal{K}_{D, Q_{12}} S^{-1} w=g_{0} \text { for } w=S T_{0, \partial Q_{12}} v \in H^{-1 / 2, o} .
$$

Now we can apply Theorem 7.9 and Theorem 7.10 to conclude:

- For the critical case $\Re e(\omega)-\frac{1}{4} \in \mathbb{Z}$ the operators $L, L_{0}, L_{0, c}, T$ are not normally solvable;

- They are simultaneously normalized replacing $T$ defined in $(7.15)$ by

$$
T^{\prec}=\operatorname{Rst} r_{+} A_{\Upsilon}: H^{-1 / 2, o} \rightarrow \widetilde{H}^{-1 / 2}\left(\mathbb{R}_{+}\right)
$$

with a restricted image and using the relations 7.15 for the other operators;

- In case of $\Re e(\omega)=\frac{1}{4}$ the problem becomes well-posed if we restrict the image space by the compatibility condition

$$
g_{0}=g_{1}-B_{1} R g_{2} \in \widetilde{H}^{-1 / 2}\left(\mathbb{R}_{+}\right) .
$$

Herein we have $B_{1}=\mathcal{F}^{-1} \sigma_{1} \cdot \mathcal{F}, \sigma_{1}=\alpha-\beta t(\xi)+\gamma \vartheta(\xi)$ and may choose a right inverse $R$ from 7.9 as

$$
R g_{2}\left(x_{1}, x_{2}\right)=\frac{1}{2 \pi} \int_{\mathbb{R}} e^{-i \xi x_{2}-t(\xi) x_{1}} \sigma_{2}^{-1}(\xi) \widehat{\ell^{o} g_{2}}(\xi) d \xi, \quad\left(x_{1}, x_{2}\right) \in Q_{1}
$$

with an odd extension of $g_{2} \in H^{-1 / 2}\left(\mathbb{R}_{+}\right)$to $\ell^{o} g_{2} \in H^{-1 / 2}(\mathbb{R})$, which allows further interpretation of the compatibility condition;

- In the critical case $\Re e(\omega)-\frac{1}{4} \in \mathbb{Z} \backslash\{0\}$ we furthermore obtain one-sided invertible operators after normalization and analogous results in spaces of higher regularity $(\varepsilon>0)$.

\section{Further results and open problems}

In this section we like to mention some possibilities of future research in continuation of the present work. The first part is devoted to straightforward ideas, the second to vague extensions, the third is directed towards open problems.

\subsection{Variants and generalizations}

The results of this paper can be generalized in various directions by analogy to existing research about related classes of operators. So matrix operators with elements in $H^{p, s}, W^{p, s}$ etc. gives a large field of possible extensions, see [6, 9. However its usefulness in applications is not yet clear.

Applications in higher dimensions such as 3D wedge diffraction problems can be tackled and are interesting from the applications' point of view as in the case of Sommerfeld diffraction problems, cf. 37.

Other BVPs can be considered where the Helmholtz equation is replaced by other elliptic PDEs or systems such as the Lamé equations [27]. 
Asymptotic of solutions can be studied as in 30 .

\subsection{Further possible connected research}

BVPs and transmission problems in domains with rectangular configuration can be reduced or are related to the present class of operators. This may help to solve multi-media problems of the kind considered in 24].

There are further forms of symmetry, defined via EAE and matrical coupling relations, which are interesting in applications and may be tackled in combination with the basic ideas of the present approach, see 2,34 .

In many cases the solution by the present method is (logically) equivalent to the (generalized) inversion of an operator matrix of the form (7.2), see [11. The corresponding solution formulas imply correlations for the Fourier integral operators, cf. formulas involving Hankel operators in 25. A systematic investigation could produce new kinds of operator matrix identities and insights from the operator theoretical point of view. See also the connected Remark 7.14.

Localization techniques [5] may be applied to tackle certain BVPs for elliptic PDEs and boundary conditions with non-constant coefficients in what concerns the Fredholm property and normalization of such problems.

\subsection{Open problems}

We end up by giving a list of consequent open problems:

- To which extend does Proposition 4.6 hold for larger symbol classes than $\mathcal{G} C^{\nu}(\ddot{\mathbb{R}})$ ?

- Is it true that the Fredholm property of $T_{0}$ implies that $\Upsilon$ is invertible? A similar result is known for Toeplitz operators and WHOs [23].

- Is there a Coburn-like lemma 4!: If $T$ is given by (1.1) and $\Upsilon$ is invertible of order $r-s$, then $T$ is one-sided invertible?

- To which extend does the shifting process transfer generalized inverses (cf. Theorem 4.5), if the symbol is not Hölder continuous?

- What about problems in cones different from $Q_{1}$, for instance rational angles, cf. [17, 18]?

\section{Acknowledgment}

The work was supported by FCT-Portuguese Foundation for Science and Technology through the Center for Research and Development in Mathematics and Applications (CIDMA) at Universidade de Aveiro, within UID/MAT/04106/2013, and the Center for Functional Analysis, Linear Structures and Applications (CEAFEL) at Instituto Superior Técnico, Universidade de Lisboa, Portugal.

\section{References}

[1] H. Bart, I. Gohberg and M. Kaashoek, The coupling method for solving integral equations. Oper. Theory Adv. Appl. 2 (1984), 39-73. 
[2] H. Bart and V.E. Tsekanovskii, Matricial coupling and equivalence after extension. Oper. Theory Adv. Appl. 59 (1991), 143-160.

[3] E.L. Basor and T. Ehrhardt, On a class of Toeplitz + Hankel operators. New York J. Math. 5 (1999), 1-16.

[4] A. Böttcher and B. Silbermann, Analysis of Toeplitz Operators. Springer, Berlin 2006.

[5] L.P. Castro, R. Duduchava and F.-O. Speck, Localization and minimal normalization of some basic mixed boundary value problems. In: Factorization, Singular Operators and Related Problems (Eds: S. Samko et al.), Kluwer, Dordrecht 2003, 73-100.

[6] L.P. Castro, R. Duduchava and F.-O. Speck, Asymmetric factorizations of matrix functions on the real line. In: Modern Operator Theory and Applications. The Igor Borisovich Simonenko Anniversary Volume (Eds: Y.M. Erusalimskii et al.), Oper. Theory Adv. Appl. 170, Birkhäuser, Basel 2006, 53-74.

[7] L.P. Castro and F.-O. Speck, On the characterization of the intermediate space in generalized factorizations. Math. Nachr. 176 (1995), 39-54.

[8] L.P. Castro and F.-O. Speck, Regularity properties and generalized inverses of deltarelated operators. Z. Anal. Anwend. 17 (1998), 577-598.

[9] L.P. Castro and F.-O. Speck, Inversion of matrix convolution type operators with symmetry. Portugaliae Mathematica 62 (2005), 193-216.

[10] L.P. Castro, F.-O. Speck and F.S. Teixeira,

On a class of wedge diffraction problems posted by Erhard Meister. Oper. Theory Adv. Appl. 147 (2004), 211-238.

[11] L.P. Castro, F.-O. Speck and F.S. Teixeira, Mixed boundary value problems for the Helmholtz equation in a quadrant. Integr. Equ. Oper. Theory 56 (2006), 1-44.

[12] A. Devinatz and M. Shinbrot, General Wiener-Hopf operators. Trans. AMS $\mathbf{1 4 5}$ (1969), 467-494.

[13] R. Duduchava, Integral Equations with Fixed Singularities. Teubner, Leipzig 1979.

[14] R. Duduchava and F.-O. Speck, Bessel potential operators for the quarter-plane. Appl. Anal. 45 (1992), 49-68.

[15] R. Duduchava and F.-O. Speck, Pseudodifferential operators on compact manifolds with Lipschitz boundary. Math. Nachr. 160 (1993), 149-191.

[16] T. Ehrhardt, Invertibility theory for Toeplitz plus Hankel operators and singular integral operators with flip. J. Funct. Anal. 208 (2004), 64-106.

[17] T. Ehrhardt, A.P. Nolasco and F.-O. Speck, Boundary integral methods for wedge diffraction problems: the angle $2 \pi / n$, Dirichlet and Neumann conditions. Oper. Matrices 5 (2011), 1-40.

[18] T. Ehrhardt, A.P. Nolasco and F.-O. Speck, A Riemann surface approach for diffraction from rational angles. Oper. Matrices 8 (2014), 301-355.

[19] G.I. Eskin, Boundary Value Problems for Elliptic Pseudodifferential Equations. American Mathematical Society, Providence, Rhode Island, 1981 (Russian edition 1973).

[20] P. Grisvard, Elliptic Problems in Non-Smooth Domains. Pitman, London, 1985. 
[21] S. ter Horst and A.C.M. Ran, Equivalence after extension and matricial coupling coincide with Schur coupling, on separable Hilbert spaces. Linear Algebra Appl. 439 (2013), 793-805.

[22] G.C. Hsiao and W.L. Wendland, Boundary Integral Equations. Springer, Berlin, 2008.

[23] G.S. Litvinchuk and I.M. Spitkovsky, Factorization of Measurable Matrix Functions. Oper. Theory Adv. Appl. 25, Birkhäuser, Basel 1987.

[24] E. Meister, Some solved and unsolved canonical problems of diffraction theory. Differential Equations and their Applications, Proc. 6th Int. Conf. Equadiff at Brno/Czech. 1985, Lect. Notes Math. 1192 (Springer, Berlin, 1986), 393-398.

[25] E. Meister, F. Penzel, F.-O. Speck and F.S. Teixeira, Some interior and exterior boundary value problems for the Helmholtz equation in a quadrant. Proc. R. Soc. Edinb., Sect. A 123 (1993), 275-294.

[26] E. Meister and F.-O. Speck, Modern Wiener-Hopf methods in diffraction theory. In: Ordinary and Partial Differential Equations 2, Pitman Res. Notes Math. Ser. 216 (Longman, London, 1989), 130-171.

[27] E. Meister and F.-O. Speck, The explicit solution of elastodynamical diffraction problems by symbol factorization. Z. Anal. Anw. 8 (1989), 307-328.

[28] A. Moura Santos, F.-O. Speck and F.S. Teixeira, Compatibility conditions in some diffraction problems. In: Direct and inverse electromagnetic scattering. Pitman Res. Notes Math. Ser. 361 (Longman, London 1996), 25-38.

[29] A. Moura Santos, F.-O. Speck and F.S. Teixeira, Minimal normalization of WienerHopf operators in spaces of Bessel potentials. J. Math. Anal. Appl. 225 (1998), 501531.

[30] F. Penzel and F.-O. Speck, Asymptotic expansion of singular operators in Sobolev spaces. Asymptotic Analysis 7 (1993), 287-300.

[31] I.B. Simonenko, Some general questions in the theory of the Riemann boundary problem. Izv. Akad. Nauk SSSR, Ser. Mat. 32 (1968), 1138-1146 (in Russian). Math. USSR, Izv. 2 (1970), 1091-1099 (in English).

[32] F.-O. Speck, General Wiener-Hopf Factorization Methods. Pitman, London, 1985.

[33] F.-O. Speck, Mixed boundary value problems of the type of Sommerfeld's half-plane problem. Proc. R. Soc. Edinb., Sect. A 104 (1986), 261-277.

[34] F.-O. Speck, Diffraction by a three-quarter-plane using an abstract Babinet principle. Z. Angew. Math. Mech. 93 (2012), 485-491.

[35] F.-O. Speck, On the reduction of linear systems related to boundary value problems. In: Operator Theory, Pseudo-Differential Equations, and Mathematical Physics. The Vladimir Rabinovich Anniversary Volume, Oper. Theory Adv. Appl. 228 (Eds. Y.I. Karlovich, L. Rodino, B. Silbermann and I.M. Spitkovsky) Birkhäuser, Berlin (2013), 391-406.

[36] F.-O. Speck, Wiener-Hopf factorization through an intermediate space. Integr. Equ. Oper. Theory 82 (2015), 395-415.

[37] F.-O. Speck, A class of interface problems for the Helmholtz equation in $\mathbb{R}^{n}$. Math. Meth. Appl. Sciences 40 (2017), 391-403. 
[38] D. Timotin, Schur coupling and related equivalence relations for operators on a Hilbert space. Linear Algebra Appl. 452 (2014), 106-119.

Luís Pinheiro de Castro

CIDMA and Departamento de Matemática

Universidade de Aveiro

Aveiro, Portugal

e-mail: castro@ua.pt

Frank-Olme Speck

Instituto Superior Técnico

Universidade de Lisboa

Avenida Rovisco Pais

1049-001 Lisboa, Portugal

e-mail: fspeck@tecnico.pt 